\title{
ON THE INTERPLAY BETWEEN MEASURABLE AND TOPOLOGICAL DYNAMICS
}

\author{
E. GLASNER AND B. WEISS
}

Contents

Introduction 2

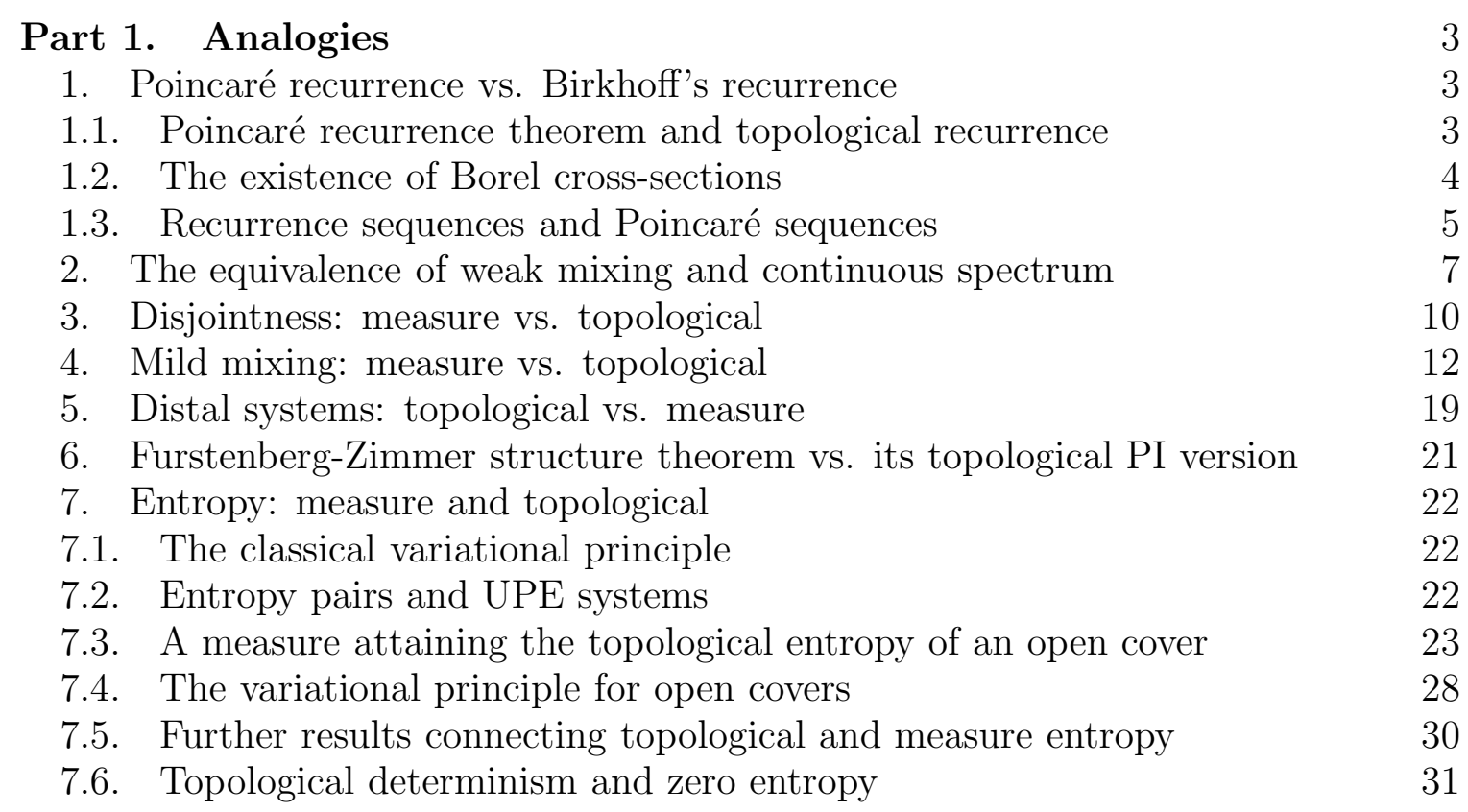

\begin{tabular}{lll}
\hline Part 2. Meeting grounds & 33
\end{tabular}

8. Unique ergodicitv 33

9. The relative Jewett-Krieger theorem 34

10. Models for other commutative diagrams 39

11. The Furstenberg-Weiss almost 1-1 extension theorem 40

12. Cantor minimal representations 40

13. Other related theorems 41

References 44 


\section{INTRODUCTION}

Recurrent - wandering, conservative - dissipative, contracting - expanding, deterministic - chaotic, isometric - mixing, periodic - turbulent, distal - proximal, the list can go on and on. These (pairs of) words - all of which can be found in the dictionary - convey dynamical images and were therefore adopted by mathematicians to denote one or another mathematical aspect of a dynamical system.

The two sister branches of the theory of dynamical systems called ergodic theory (or measurable dynamics) and topological dynamics use these words to describe different but parallel notions in their respective theories and the surprising fact is that many of the corresponding results are rather similar. In the following article we have tried to demonstrate both the parallelism and the discord between ergodic theory and topological dynamics. We hope that the subjects we chose to deal with will successfully demonstrate this duality.

The table of contents gives a detailed listing of the topics covered. In the first part we have detailed the strong analogies between ergodic theory and topological dynamics as shown in the treatment of recurrence phenomena, equicontinuity and weak mixing, distality and entropy. In the case of distality the topological version came first and the theory of measurable distality was strongly influenced by the topological results. For entropy theory the influence clearly was in the opposite direction. The prototypical result of the second part is the statement that any abstract measure probability preserving system can be represented as a continuous transformation of a compact space, and thus in some sense ergodic theory embeds into topological dynamics.

We have not attempted in any way to be either systematic or comprehensive. Rather our choice of subjects was motivated by taste, interest and knowledge and to great extent is random. We did try to make the survey accessible to non-specialists, and for this reason we deal throughout with the simplest case of actions of $\mathbb{Z}$. Most of the discussion carries over to noninvertible mappings and to $\mathbb{R}$ actions. Indeed much of what we describe can be carried over to general amenable groups. Similarly, we have for the most part given rather complete definitions. Nonetheless, we did take advantage of the fact that this article is part of a handbook and for some of the definitions, basic notions and well known results we refer the reader to the earlier introductory chapters of volume I. Finally, we should acknowledge the fact that we made use of parts of our previous expositions [86] and [35].

We made the writing of this survey more pleasurable for us by the introduction of a few original results. In particular the following results are entirely or partially new. Theorem 1.2 (the equivalence of the existence of a Borel cross-section with the coincidence of recurrence and periodicity), most of the material in Section 4 (on topological mild-mixing), all of subsection 7.4 (the converse side of the local variational principle) and subsection 7.6 (on topological determinism). 


\section{Part 1. Analogies}

\section{Poincaré ReCurrence Vs. Birkhoff's ReCurrence}

1.1. Poincaré recurrence theorem and topological recurrence. The simplest dynamical systems are the periodic ones. In the absence of periodicity the crudest approximation to this is approximate periodicity where instead of some iterate $T^{n} x$ returning exactly to $x$ it returns to a neighborhood of $x$. The first theorem in abstract measure dynamics is Poincaré's recurrence theorem which asserts that for a finite measure preserving system $(X, \mathcal{B}, \mu, T)$ and any measurable set $A, \mu$-a.e. point of $A$ returns to $A$ (see [46, Theorem 4.3.1]). The proof of this basic fact is rather simple and depends on identifying the set of points $W \subset A$ that never return to $A$. These are called the wandering points and their measurability follows from the formula

$$
W=A \cap\left(\bigcap_{k=1}^{\infty} T^{-k}(X \backslash A)\right) .
$$

Now for $n \geq 0$, the sets $T^{-n} W$ are pairwise disjoint since $x \in T^{-n} W$ means that the forward orbit of $x$ visits $A$ for the last time at moment $n$. Since $\mu\left(T^{-n} W\right)=\mu(W)$ it follows that $\mu(W)=0$ which is the assertion of Poincaré's theorem. Noting that $A \cap T^{-n} W$ describes the points of $A$ which visit $A$ for the last time at moment $n$, and that $\mu\left(\cup_{n=0}^{\infty} T^{-n} W\right)=0$ we have established the following stronger formulation of Poincaré's theorem.

1.1. Theorem. For a finite measure preserving system $(X, \mathcal{B}, \mu, T)$ and any measurable set $A, \mu$-a.e. point of $A$ returns to $A$ infinitely often.

Note that only sets of the form $T^{-n} B$ appeared in the above discussion so that the invertibility of $T$ is not needed for this result. In the situation of classical dynamics, which was Poincaré's main interest, $X$ is also equipped with a separable metric topology. In such a situation we can apply the theorem to a refining sequence of partitions $\mathcal{P}_{m}$, where each $\mathcal{P}_{m}$ is a countable partition into sets of diameter at most $\frac{1}{m}$. Applying the theorem to a fixed $\mathcal{P}_{m}$ we see that $\mu$-a.e. point comes to within $\frac{1}{m}$ of itself, and since the intersection of a sequence of sets of full measure has full measure, we deduce the corollary that $\mu$-a.e. point of $X$ is recurrent.

This is the measure theoretical path to the recurrence phenomenon which depends on the presence of a finite invariant measure. The necessity of such measure is clear from considering translation by one on the integers. The system is dissipative, in the sense that no recurrence takes place even though there is an infinite invariant measure.

There is also a topological path to recurrence which was developed in an abstract setting by G. D. Birkhoff. Here the above example is eliminated by requiring that the topological space $X$, on which our continuous transformation $T$ acts, be compact. It is possible to show that in this setting a finite $T$-invariant measure always exists, and so we can retrieve the measure theoretical picture, but a purely topological discussion will give us better insight. 
A key notion here is that of minimality. A nonempty closed, $T$-invariant set $E \subset X$, is said to be minimal if $F \subset E$, closed and $T$-invariant implies $F=\emptyset$ or $F=E$. If $X$ itself is a minimal set we say that the system $(X, T)$ is a minimal system.

Fix now a point $x_{0} \in X$ and consider

$$
\omega\left(x_{0}\right)=\bigcap_{n=1}^{\infty} \overline{\left\{T^{k} x_{0}: k \geq n\right\}} .
$$

The points of $\omega\left(x_{0}\right)$ are called $\omega$-limit points of $x_{0},(\omega=$ last letter of the Greek alphabet) and in the separable case $y \in \omega\left(x_{0}\right)$ if and only if there is some sequence $k_{i} \rightarrow \infty$ such that $T^{k_{i}} x_{0} \rightarrow y$. If $x_{0} \in \omega\left(x_{0}\right)$ then $x_{0}$ is called a positively recurrent point.

Clearly $\omega\left(x_{0}\right)$ is a closed and $T$-invariant set. Therefore, in any nonempty minimal set $E$, any point $x_{0} \in E$ satisfies $x_{0} \in \omega\left(x_{0}\right)$ and thus we see that minimal sets have recurrent points.

In order to see that compact systems $(X, T)$ have recurrent points it remains to show that minimal sets always exist. This is an immediate consequence of Zorn's lemma applied to the family of nonempty closed $T$-invariant subsets of $X$. A slightly more constructive proof can be given when $X$ is a compact and separable metric space. One can then list a sequence of open sets $U_{1}, U_{2}, \ldots$ which generate the topology, and perform the following algorithm:

1. set $X_{0}=X$,

2. for $i=1,2, \ldots$,

$$
\text { if } \bigcup_{n=-\infty}^{\infty} T^{-n} U_{i} \supset X_{i-1} \text { put } X_{i}=X_{i-1} \text {, else put } X_{i}=X_{i-1} \backslash \bigcup_{n=-\infty}^{\infty} T^{-n} U_{i} \text {. }
$$

Note that $X_{i} \neq \emptyset$ and closed and thus $X_{\infty}=\bigcap_{i=0}^{\infty} X_{i}$ is nonempty. It is clearly $T$-invariant and for any $U_{i}$, if $U_{i} \cap X_{\infty} \neq \emptyset$ then $\bigcup_{-\infty}^{\infty} T^{-n}\left(U_{i} \cap X_{\infty}\right)=X_{\infty}$, which shows that $\left(X_{\infty}, T\right)$ is minimal.

1.2. The existence of Borel cross-sections. There is a deep connection between recurrent points in the topological context and ergodic theory. To see this we must consider quasi-invariant measures. For these matters it is better to enlarge the scope and deal with continuous actions of $\mathbb{Z}$, generated by $T$, on a complete separable metric space $X$. A probability measure $\mu$ defined on the Borel subsets of $X$ is said to be quasi-invariant if $T \cdot \mu \sim \mu$. Define such a system $(X, \mathcal{B}, \mu, T)$ to be conservative if for any measurable set $A, T A \subset A$ implies $\mu(A \backslash T A)=0$.

It is not hard to see that the conclusion of Poincaré's recurrence theorem holds for such systems; i.e. if $\mu(A)>0$, then $\mu$-a.e. $x$ returns to $A$ infinitely often. Thus once again $\mu$-a.e. point is topologically recurrent. It turns out now that the existence of a single topologically recurrent point implies the existence of a non-atomic conservative quasi-invariant measure. A simple proof of this fact can be found in [56] for the case when $X$ is compact - but the proof given there is equally valid for complete separable metric spaces. In this sense the phenomenon of Poincaré recurrence and topological recurrence are "equivalent" with each implying the other.

A Borel set $B \subset X$ such that each orbit intersects $B$ in exactly one point is called a Borel cross-section for the system $(X, T)$. If a Borel cross-section exists, then no non-atomic conservative quasi-invariant measure can exist. In [82 it is shown 
that the converse is also valid - namely if there are no conservative quasi-invariant measures then there is a Borel cross-section.

Note that the periodic points of $(X, T)$ form a Borel subset for which a crosssection always exists, so that we can conclude from the above discussion the following statement in which no explicit mention is made of measures.

1.2. Theorem. For a system $(X, T)$, with $X$ a completely metrizable separable space, there exists a Borel cross-section if and only if the only recurrent points are the periodic ones.

1.3. Remark. Already in [42] as well as in [21] one finds many equivalent conditions for the existence of a Borel section for a system $(X, T)$. However one doesn't find there explicit mention of conditions in terms of recurrence. Silvestrov and Tomiyama [76] established the theorem in this formulation for $X$ compact (using $C^{*}$-algebra methods). We thank A. Lazar for drawing our attention to their paper.

1.3. Recurrence sequences and Poincaré sequences. We will conclude this section with a discussion of recurrence sequences and Poincaré sequences. First for some definitions. Let us say that $D$ is a recurrence set if for any dynamical system $(Y, T)$ with compatible metric $\rho$ and any $\epsilon>0$ there is a point $y_{0}$ and a $d \in D$ with

$$
\rho\left(T^{d} y_{0}, y_{0}\right)<\epsilon
$$

Since any system contains minimal sets it suffices to restrict attention here to minimal systems. For minimal systems the set of such $y$ 's for a fixed $\epsilon$ is a dense open set.

To see this fact, let $U$ be an open set. By the minimality there is some $N$ such that for any $y \in Y$, and some $0 \leq n \leq N$, we have $T^{n} y \in U$. Using the uniform continuity of $T^{n}$, we find now a $\delta>0$ such that if $\rho(u, v)<\delta$ then for all $0 \leq n \leq N$

$$
\rho\left(T^{n} u, T^{n} v\right)<\epsilon
$$

Now let $z_{0}$ be a point in $Y$ and $d_{0} \in D$ such that

$$
p\left(T^{d_{0}} z_{0}, z_{0}\right)<\delta .
$$

For some $0 \leq n_{0} \leq N$ we have $T^{n_{0}} z_{0}=y_{0} \in U$ and from (11) we get $\rho\left(T^{d_{0}} y_{0}, y_{0}\right)<\epsilon$. Thus points that $\epsilon$ return form an open dense set. Intersecting over $\epsilon \rightarrow 0$ gives a dense $G_{\delta}$ in $Y$ of points $y$ for which

$$
\inf _{d \in D} \rho\left(T^{d} y, y\right)=0 .
$$

Thus there are points which actually recur along times drawn from the given recurrence set.

A nice example of a recurrence set is the set of squares. To see this it is easier to prove a stronger property which is the analogue in ergodic theory of recurrence sets.

1.4. Definition. A sequence $\left\{s_{j}\right\}$ is said to be a Poincaré sequence if for any finite measure preserving system $(X, \mathcal{B}, \mu, T)$ and any $B \in \mathcal{B}$ with positive measure we have

$$
\mu\left(T^{s_{j}} B \cap B\right)>0 \quad \text { for some } s_{j} \text { in the sequence. }
$$


Since any minimal topological system $(Y, T)$ has finite invariant measures with global support, $\mu$ any Poincaré sequence is recurrence sequence. Indeed for any presumptive constant $b>0$ which would witness the non-recurrence of $\left\{s_{j}\right\}$ for $(Y, T)$, there would have to be an open set $B$ with diameter less than $b$ and having positive $\mu$-measure such that $T^{s_{j}} B \cap B$ is empty for all $\left\{s_{j}\right\}$.

Here is a sufficient condition for a sequence to be a Poincaré sequence:

1.5. Lemma. If for every $\alpha \in(0,2 \pi)$

$$
\lim _{n \rightarrow \infty} \frac{1}{n} \sum_{k=1}^{n} e^{i \alpha s_{k}}=0
$$

then $\left\{s_{k}\right\}_{1}^{\infty}$ is a Poincaré sequence.

Proof. Let $(X, \mathcal{B}, \mu, T)$ be a measure preserving system and let $U$ be the unitary operator defined on $L^{2}(X, \mathcal{B}, \mu)$ by the action of $T$, i.e.

$$
(U f)(x)=f(T x) \text {. }
$$

Let $H_{0}$ denote the subspace of invariant functions and for a set of positive measure $B$, let $f_{0}$ be the projection of $1_{B}$ on the invariant functions. Since this can also be seen as a conditional expectation with respect to the $\sigma$-algebra of invariant sets $f_{0} \geq 0$ and is not zero. Now since $\mathbf{1}_{B}-f_{0}$ is orthogonal to the space of invariant functions its spectral measure with respect to $U$ doesn't have any atoms at $\{0\}$. Thus from the spectral representation we deduce that in $L^{2}$-norm

$$
\left\|\frac{1}{n} \sum_{1}^{n} U^{s_{k}}\left(1_{B}-f_{0}\right)\right\|_{L^{2}} \longrightarrow 0
$$

or

$$
\left\|\left(\frac{1}{n} \sum_{1}^{n} U^{s_{k}} 1_{B}\right)-f_{0}\right\|_{L_{2}} \longrightarrow 0
$$

and integrating against $1_{B}$ and using the fact that $f_{0}$ is the projection of $1_{B}$ we see that

$$
\lim _{n \rightarrow \infty} \frac{1}{n} \sum_{1}^{n} \mu\left(B \cap T^{-s_{k}} B\right)=\left\|f_{0}\right\|^{2}>0
$$

which clearly implies that $\left\{s_{k}\right\}$ is a Poincaré sequence.

The proof we have just given is in fact von-Neumann's original proof for the mean ergodic theorem. He used the fact that $\mathbb{N}$ satisfies the assumptions of the proposition, which is Weyl's famous theorem on the equidistribution of $\{n \alpha\}$. Returning to the squares Weyl also showed that $\left\{n^{2} \alpha\right\}$ is equidistributed for all irrational $\alpha$. For rational $\alpha$ the exponential sum in the lemma needn't vanish, however the recurrence along squares for the rational part of the spectrum is easily verified directly so that we can conclude that indeed the squares are a Poincaré sequence and hence a recurrence sequence.

The converse is not always true, i.e. there are recurrence sequences that are not Poincaré sequences. This was first shown by I. Kriz [60] in a beautiful example (see also [86, Chapter 5]). Finally here is a simple problem. 
Problem: If $D$ is a recurrence sequence for all circle rotations is it a recurrence set?

A little bit of evidence for a positive answer to that problem comes from looking at a slightly different characterization of recurrence sets. Let $\mathcal{N}$ denote the collection of sets of the form

$$
N(U, U)=\left\{n: T^{-n} U \cap U \neq \emptyset\right\}, \quad \text { (U open and nonempty), }
$$

where $T$ is a minimal transformation. Denote by $\mathcal{N}^{*}$ the subsets of $\mathbb{N}$ that have a nonempty intersection with every element of $\mathcal{N}$. Then $\mathcal{N}^{*}$ is exactly the class of recurrence sets. For minimal transformations, another description of $N(U, U)$ is obtained by fixing some $y_{0}$ and denoting

$$
N\left(y_{0}, U\right)=\left\{n: T^{n} y_{0} \in U\right\}
$$

Then $N(U, U)=N\left(y_{0}, U\right)-N\left(y_{0}, U\right)$. Notice that the minimality of $T$ implies that $N\left(y_{0}, U\right)$ is a syndetic set (a set with bounded gaps) and so any $N(U, U)$ is the set of differences of a syndetic set. Thus $\mathcal{N}$ consists essentially of all sets of the form $S-S$ where $S$ is a syndetic set.

Given a finite set of real numbers $\left\{\lambda_{1}, \lambda_{2}, \ldots, \lambda_{k}\right\}$ and $\epsilon>0$ set

$$
V\left(\lambda_{1}, \lambda_{2}, \ldots, \lambda_{k} ; \epsilon\right)=\left\{n \in \mathbb{Z}: \max _{j}\left\{\left\|n \lambda_{j}\right\|<\epsilon\right\}\right\},
$$

where $\|\cdot\|$ denotes the distance to the closest integer. The collection of such sets forms a basis of neighborhoods at zero for a topology on $\mathbb{Z}$ which makes it a topological group. This topology is called the Bohr topology. (The corresponding uniform structure is totally bounded and the completion of $\mathbb{Z}$ with respect to it is a compact topological group called the Bohr compactification of $\mathbb{Z}$.)

Veech proved in 78 , that any set of the form $S-S$ with $S \subset \mathbb{Z}$ syndetic contains a neighborhood of zero in the Bohr topology up to a set of zero density. It is not known if in that statement the zero density set can be omitted. If it could then a positive answer to the above problem would follow (see also [32]).

\section{The EQUivalence OF WeAK MiXing AND CONTINUOUS SPECTRUm}

In order to analyze the structure of a dynamical system $\mathbf{X}$ there are, a priori, two possible approaches. In the first approach one considers the collection of subsystems $Y \subset X$ (i.e. closed $T$-invariant subsets) and tries to understand how $X$ is built up by these subsystems. In the other approach one is interested in the collection of factors $X \stackrel{\pi}{\rightarrow} Y$ of the system $\mathbf{X}$. In the measure theoretical case the first approach leads to the ergodic decomposition and thereby to the study of the "indecomposable" or ergodic components of the system. In the topological setup there is, unfortunately, no such convenient decomposition describing the system in terms of its indecomposable parts and one has to use some less satisfactory substitutes. Natural candidates for indecomposable components of a topological dynamical system are the "orbit closures" (i.e. the topologically transitive subsystems) or the "prolongation" cells (which often coincide with the orbit closures), see [4. The minimal subsystems are of particular importance here. Although we can not say, in any reasonable sense, that the study of the general system can be reduced to that of its minimal components, the analysis of 
the minimal systems is nevertheless an important step towards a better understanding of the general system.

This reasoning leads us to the study of the collection of indecomposable systems (ergodic systems in the measure category and transitive or minimal systems in the topological case) and their factors. The simplest and best understood indecomposable dynamical systems are the ergodic translations of a compact monothetic group (a cyclic permutation on $\mathbb{Z}_{p}$ for a prime number $p$, the "adding machine" on $\prod_{n=0}^{\infty} \mathbb{Z}_{2}$, an irrational rotation $z \mapsto e^{2 \pi i \alpha} z$ on $S^{1}=\{z \in \mathbb{C}:|z|=1\}$ etc.). It is not hard to show that this class of ergodic actions is characterized as those dynamical systems which admit a model $(X, X, \mu, T)$ where $X$ is a compact metric space, $T: X \rightarrow$ $X$ a surjective isometry and $\mu$ is $T$-ergodic. We call these systems Kronecker or isometric systems. Thus our first question concerning the existence of factors should be: given an ergodic dynamical system $\mathbf{X}$ which are its Kronecker factors? Recall that a measure dynamical system $\mathbf{X}=(X, X, \mu, T)$ is called weakly mixing if the product system $(X \times X, X \otimes X, \mu \times \mu, T \times T)$ is ergodic. The following classical theorem is due to von Neumann. The short and elegant proof we give was suggested by Y. Katznelson.

2.1. Theorem. An ergodic system $\mathbf{X}$ is weakly mixing iff it admits no nontrivial Kronecker factor.

Proof. Suppose $\mathbf{X}$ is weakly mixing and admits an isometric factor. Now a factor of a weakly mixing system is also weakly mixing and the only system which is both isometric and weakly mixing is the trivial system (an easy exercise). Thus a weakly mixing system does not admit a nontrivial Kronecker factor.

For the other direction, if $\mathbf{X}$ is non-weakly mixing then in the product space $X \times X$ there exists a $T$-invariant measurable subset $W$ such that $0<(\mu \times \mu)(W)<1$. For every $x \in X$ let $W(x)=\left\{x^{\prime} \in X:\left(x, x^{\prime}\right) \in W\right\}$ and let $f_{x}=\mathbf{1}_{W(x)}$, a function in $L^{\infty}(\mu)$. It is easy to check that $U_{T} f_{x}=f_{T^{-1} x}$ so that the map $\pi: X \rightarrow L^{2}(\mu)$ defined by $\pi(x)=f_{x}, x \in X$ is a Borel factor map. Denoting

$$
\pi(X)=Y \subset L^{2}(\mu), \quad \text { and } \quad \nu=\pi_{*}(\mu),
$$

we now have a factor map $\pi: \mathbf{X} \rightarrow(Y, \nu)$. Now the function $\|\pi(x)\|$ is clearly measurable and invariant and by ergodicity it is a constant $\mu$-a.e.; say $\|\pi(x)\|=1$. The dynamical system $(Y, \nu)$ is thus a subsystem of the compact dynamical system $\left(B, U_{T}\right)$, where $B$ is the unit ball of the Hilbert space $L^{2}(\mu)$ and $U_{T}$ is the Koopman unitary operator induced by $T$ on $L^{2}(\mu)$. Now it is well known (see e.g. 35]) that a compact topologically transitive subsystem which carries an invariant probability measure must be a Kronecker system and our proof is complete.

Concerning the terminology we used in the proof of Theorem 2.1. B. O. Koopman, a student of G. D. Birkhoff and a co-author of both Birkhoff and von Neumann introduced the crucial idea of associating with a measure dynamical system $\mathbf{X}=$ $(X, X, \mu, T)$ the unitary operator $U_{T}$ on the Hilbert space $L^{2}(\mu)$. It is now an easy matter to see that Theorem 2.1 can be re-formulated as saying that the system $\mathbf{X}$ is weakly mixing iff the point spectrum of the Koopman operator $U_{T}$ comprises the single complex number 1 with multiplicity 1 . Or, put otherwise, that the one dimensional space of constant functions is the eigenspace corresponding to the eigenvalue 1 (this 
fact alone is equivalent to the ergodicity of the dynamical system) and that the restriction of $U_{T}$ to the orthogonal complement of the space of constant functions has a continuous spectrum.

We now consider a topological analogue of this theorem. Recall that a topological system $(X, T)$ is topologically weakly mixing when the product system $(X \times X, T \times T)$ is topologically transitive. It is equicontinuous when the family $\left\{T^{n}: n \in \mathbb{Z}\right\}$ is an equicontinuous family of maps. Again an equivalent condition is the existence of a compatible metric with respect to which $T$ is an isometry. And, moreover, a minimal system is equicontinuous iff it is a minimal translation on a compact monothetic group. We will need the following lemma.

2.2. Lemma. Let $(X, T)$ be a minimal system and $f: X \rightarrow \mathbb{R}$ a T-invariant function with at least one point of continuity (for example this is the case when $f$ is lower or upper semi-continuous or more generally when it is the pointwise limit of a sequence of continuous functions), then $f$ is a constant.

Proof. Let $x_{0}$ be a continuity point and $x$ an arbitrary point in $X$. Since $\left\{T^{n} x\right.$ : $n \in \mathbb{Z}\}$ is dense and as the value $f\left(T^{n} x\right)$ does not depend on $n$ it follows that $f(x)=f\left(x_{0}\right)$.

2.3. Theorem. Let $(X, T)$ be a minimal system then $(X, T)$ is topologically weakly mixing iff it has no non-trivial equicontinuous factor.

Proof. Suppose $(X, T)$ is minimal and topologically weakly mixing and let $\pi:(X, T) \rightarrow$ $(Y, T)$ be an equicontinuous factor. If $\left(x, x^{\prime}\right)$ is a point whose $T \times T$ orbit is dense in $X \times X$ then $\left(y, y^{\prime}\right)=\left(\pi(x), \pi\left(x^{\prime}\right)\right)$ has a dense orbit in $Y \times Y$. However, if $(Y, T)$ is equicontinuous then $Y$ admits a compatible metric with respect to which $T$ is an isometry and the existence of a transitive point in $Y \times Y$ implies that $Y$ is a trivial one point space.

Conversely, assuming that $(X \times X, T \times T)$ is not transitive we will construct an equicontinuous factor $(Z, T)$ of $(X, T)$. As $(X, T)$ is a minimal system, there exists a $T$-invariant probability measure $\mu$ on $X$ with full support. By assumption there exists an open $T$-invariant subset $U$ of $X \times X$, such that cls $U:=M \subsetneq X \times X$. By minimality the projections of $M$ to both $X$ coordinates are onto. For every $y \in X$ let $M(y)=\{x \in X:(x, y) \in M\}$, and let $f_{y}=\mathbf{1}_{M(y)}$ be the indicator function of the set $M(y)$, considered as an element of $L^{1}(X, \mu)$.

Denote by $\pi: X \rightarrow L^{1}(X, \mu)$ the map $y \mapsto f_{y}$. We will show that $\pi$ is a continuous homomorphism, where we consider $L^{1}(X, \mu)$ as a dynamical system with the isometric action of the group $\left\{U_{T}^{n}: n \in \mathbb{Z}\right\}$ and $U_{T} f(x)=f(T x)$. Fix $y_{0} \in X$ and $\epsilon>0$. There exists an open neighborhood $V$ of the closed set $M\left(y_{0}\right)$ with $\mu\left(V \backslash M\left(y_{0}\right)\right)<\epsilon$. Since $M$ is closed the set map $y \mapsto M(y), X \rightarrow 2^{X}$ is upper semi-continuous and we can find a neighborhood $W$ of $y_{0}$ such that $M(y) \subset V$ for every $y \in W$. Thus for every $y \in W$ we have $\mu\left(M(y) \backslash M\left(y_{0}\right)\right)<\epsilon$. In particular, $\mu(M(y)) \leq \mu\left(M\left(y_{0}\right)\right)+\epsilon$ and it follows that the map $y \mapsto \mu(M(y))$ is upper semi-continuous. A simple computation shows that it is $T$-invariant, hence, by Lemma 2.2, a constant. 
With $y_{0}, \epsilon$ and $V, W$ as above, for every $y \in W, \mu\left(M(y) \backslash M\left(y_{0}\right)\right)<\epsilon$ and $\mu(M(y))=$ $\mu\left(M\left(y_{0}\right)\right)$, thus $\mu\left(M(y) \Delta M\left(y_{0}\right)\right)<2 \epsilon$, i.e., $\left\|f_{y}-f_{y_{0}}\right\|_{1}<2 \epsilon$. This proves the claim that $\pi$ is continuous.

Let $Z=\pi(X)$ be the image of $X$ in $L^{1}(\mu)$. Since $\pi$ is continuous, $Z$ is compact. It is easy to see that the $T$-invariance of $M$ implies that for every $n \in \mathbb{Z}$ and $y \in X$, $f_{T^{-n} y}=f_{y} \circ T^{n}$ so that $Z$ is $U_{T^{-}}$invariant and $\pi:(Y, T) \rightarrow\left(Z, U_{T}\right)$ is a homomorphism. Clearly $\left(Z, U_{T}\right)$ is minimal and equicontinuous (in fact isometric).

Theorem 2.3 is due to Keynes and Robertson [57] who developed an idea of Furstenberg, 22]; and independently to K. Petersen [70] who utilized a previous work of W. A. Veech, [78. The proof we presented is an elaboration of a work of McMahon [66] due to Blanchard, Host and Maass, [13]. We take this opportunity to point out a curious phenomenon which recurs again and again. Some problems in topological dynamics - like the one we just discussed - whose formulation is purely topological, can be solved using the fact that a $\mathbb{Z}$ dynamical system always carries an invariant probability measure, and then employing a machinery provided by ergodic theory. In several cases this approach is the only one presently known for solving the problem. In the present case however purely topological proofs exist, e.g. the Petersen-Veech proof is one such.

\section{Disjointness: MeAsure Vs. TOPOlOGiCAL}

In the ring of integers $\mathbb{Z}$ two integers $m$ and $n$ have no common factor if whenever $k \mid m$ and $k \mid n$ then $k= \pm 1$. They are disjoint if $m \cdot n$ is the least common multiple of $m$ and $n$. Of course in $\mathbb{Z}$ these two notions coincide. In his seminal paper of 1967 [23], H. Furstenberg introduced the same notions in the context of dynamical systems, both measure-preserving transformations and homeomorphisms of compact spaces, and asked whether in these categories as well the two are equivalent. The notion of a factor in, say the measure category, is the natural one: the dynamical system $\mathbf{Y}=(Y, y, \nu, T)$ is a factor of the dynamical system $\mathbf{X}=(X, X, \mu, T)$ if there exists a measurable map $\pi: X \rightarrow Y$ with $\pi(\mu)=\nu$ that $T \circ \pi=\pi \circ T$. A common factor of two systems $\mathbf{X}$ and $\mathbf{Y}$ is thus a third system $\mathbf{Z}$ which is a factor of both. A joining of the two systems $\mathbf{X}$ and $\mathbf{Y}$ is any system $\mathbf{W}$ which admits both as factors and is in turn spanned by them. According to Furstenberg's definition the systems $\mathbf{X}$ and $\mathbf{Y}$ are disjoint if the product system $\mathbf{X} \times \mathbf{Y}$ is the only joining they admit. In the topological category, a joining of $(X, T)$ and $(Y, S)$ is any subsystem $W \subset X \times Y$ of the product system $(X \times Y, T \times S)$ whose projections on both coordinates are full; i.e. $\pi_{X}(W)=X$ and $\pi_{Y}(W)=Y$. $(X, T)$ and $(Y, S)$ are disjoint if $X \times Y$ is the unique joining of these two systems. It is easy to verify that if $(X, T)$ and $(Y, S)$ are disjoint then at least one of them is minimal. Also, if both systems are minimal then they are disjoint iff the product system $(X \times Y, T \times S)$ is minimal.

In 1979, D. Rudolph, using joining techniques, provided the first example of a pair of ergodic measure preserving transformations with no common factor which are not disjoint [72. In this work Rudolph laid the foundation of joining theory. He introduced the class of dynamical systems having "minimal self-joinings" (MSJ), and constructed a rank one mixing dynamical system having minimal self-joinings of all orders. 
Given a dynamical system $\mathbf{X}=(X, X, \mu, T)$ a probability measure $\lambda$ on the product of $k$ copies of $X$ denoted $X_{1}, X_{2}, \ldots, X_{k}$, invariant under the product transformation and projecting onto $\mu$ in each coordinate is a $k$-fold self-joining. It is called an off-diagonal if it is a "graph" measure of the form $\lambda=\operatorname{gr}\left(\mu, T^{n_{1}}, \ldots, T^{n_{k}}\right)$, i.e. $\lambda$ is the image of $\mu$ under the map $x \mapsto\left(T^{n_{1}} x, T^{n_{2}} x, \ldots, T^{n_{k}} x\right)$ of $X$ into $\prod_{i=1}^{k} X_{i}$. The joining $\lambda$ is a product of off-diagonals if there exists a partition $\left(J_{1}, \ldots, J_{m}\right)$ of $\{1, \ldots, k\}$ such that (i) For each $l$, the projection of $\lambda$ on $\prod_{i \in J_{l}} X_{i}$ is an off-diagonal, (ii) The systems $\prod_{i \in J_{l}} X_{i}, 1 \leq l \leq m$, are independent. An ergodic system $\mathbf{X}$ has minimal self-joinings of order $k$ if every $k$-fold ergodic self-joining of $\mathbf{X}$ is a product of off-diagonals.

In 72 Rudolph shows how any dynamical system with MSJ can be used to construct a counter example to Furstenberg's question as well as a wealth of other counter examples to various questions in ergodic theory. In [52 del Junco, Rahe and Swanson were able to show that the classical example of Chacón [16] has MSJ, answering a question of Rudolph whether a weakly but not strongly mixing system with MSJ exists. In 38 Glasner and Weiss provide a topological counterexample, which also serves as a natural counterexample in the measure category. The example consists of two horocycle flows which have no nontrivial common factor but are nevertheless not disjoint. It is based on deep results of Ratner [71] which provide a complete description of the self joinings of a horocycle flow. More recently an even more striking example was given in the topological category by E. Lindenstrauss, where two minimal dynamical systems with no nontrivial factor share a common almost 1-1 extension, 63.

Beginning with the pioneering works of Furstenberg and Rudolph, the notion of joinings was exploited by many authors; Furstenberg 1977 [24], Rudolph 1979 [72, Veech 1982 [81, Ratner 1983 [71, del Junco and Rudolph 1987 [53, Host 1991 [47, King 1992 [58, Glasner, Host and Rudolph 1992 [36], Thouvenot 1993 [77, Ryzhikov 1994 [73], Kammeyer and Rudolph 1995 (2002) [55], del Junco, Lemańczyk and Mentzen 1995 [51, and Lemańczyk, Parreau and Thouvenot 2000 [62, to mention a few. The negative answer to Furstenberg's question and the consequent works on joinings and disjointness show that in order to study the relationship between two dynamical systems it is necessary to know all the possible joinings of the two systems and to understand the nature of these joinings.

Some of the best known disjointness relations among families of dynamical systems are the following:

- id $\perp$ ergodic,

- distal $\perp$ weakly mixing ([23]),

- rigid $\perp$ mild mixing ([27]),

- zero entropy $\perp K$-systems $([23])$,

in the measure category and

- F-systems $\perp$ minimal $([23])$,

- minimal distal $\perp$ weakly mixing,

- minimal zero entropy $\perp$ minimal UPE-systems ([9]), 
in the topological category.

\section{Mild mixing: MeAsure Vs. TOPOlOGiCAL}

4.1. Definition. Let $\mathbf{X}=(X, X, \mu, T)$ be a measure dynamical system.

1. The system $\mathbf{X}$ is rigid if there exists a sequence $n_{k} \nearrow \infty$ such that

$$
\lim \mu\left(T^{n_{k}} A \cap A\right)=\mu(A)
$$

for every measurable subset $A$ of $X$. We say that $\mathbf{X}$ is $\left\{n_{k}\right\}$-rigid.

2. An ergodic system is mildly mixing if it has no non-trivial rigid factor.

These notions were introduced in [27]. The authors show that the mild mixing property is equivalent to the following multiplier property.

4.2. Theorem. An ergodic system $\mathbf{X}=(X, X, \mu, T)$ is mildly mixing iff for every ergodic (finite or infinite) measure preserving system $(Y, \mathcal{Y}, \nu, T)$, the product system

$$
(X \times Y, \mu \times \nu, T \times T),
$$

is ergodic.

Since every Kronecker system is rigid it follows from Theorem 2.1 that mild mixing implies weak mixing. Clearly strong mixing implies mild mixing. It is not hard to construct rigid weakly mixing systems, so that the class of mildly mixing systems is properly contained in the class of weakly mixing systems. Finally there are mildly but not strongly mixing systems; e.g. Chacón's system is an example (see Aaronson and Weiss [1]).

We also have the following analytic characterization of mild mixing.

\subsection{Proposition. An ergodic system $\mathbf{X}$ is mildly mixing iff}

$$
\limsup _{n \rightarrow \infty} \phi_{f}(n)<1
$$

for every matrix coefficient $\phi_{f}$, where for $f \in L^{2}(X, \mu),\|f\|=1, \phi_{f}(n):=\left\langle U_{T^{n}} f, f\right\rangle$.

Proof. If $\mathbf{X} \rightarrow \mathbf{Y}$ is a rigid factor, then there exists a sequence $n_{i} \rightarrow \infty$ such that $U_{T^{n_{i}}} \rightarrow$ id strongly on $L^{2}(Y, \nu)$. For any function $f \in L_{0}^{2}(Y, \nu)$ with $\|f\|=1$, we have $\lim _{i \rightarrow \infty} \phi_{f}\left(n_{i}\right)=1$. Conversely, if $\lim _{i \rightarrow \infty} \phi_{f}\left(n_{i}\right)=1$ for some $n_{i} \nearrow \infty$ and $f \in L_{0}^{2}(X, \mu),\|f\|=1$, then $\lim _{i \rightarrow \infty} U_{T^{n_{i}}} f=f$. Clearly $f$ can be replaced by a bounded function and we let $A$ be the sub-algebra of $L^{\infty}(X, \mu)$ generated by $\left\{U_{T^{n}} f: n \in \mathbb{Z}\right\}$. The algebra $A$ defines a non-trivial factor $\mathbf{X} \rightarrow \mathbf{Y}$ such that $U_{T^{n_{i}}} \rightarrow$ id strongly on $L^{2}(Y, \nu)$.

We say that a collection $\mathcal{F}$ of nonempty subsets of $\mathbb{Z}$ is a family if it is hereditary upward and proper (i.e. $A \subset B$ and $A \in \mathcal{F}$ implies $B \in \mathcal{F}$, and $\mathcal{F}$ is neither empty nor all of $2^{\mathbb{Z}}$ ).

With a family $\mathcal{F}$ of nonempty subsets of $\mathbb{Z}$ we associate the dual family

$$
\mathcal{F}^{*}=\{E: E \cap F \neq \emptyset, \forall F \in \mathcal{F}\} \text {. }
$$

It is easily verified that $\mathcal{F}^{*}$ is indeed a family. Also, for families, $\mathcal{F}_{1} \subset \mathcal{F}_{2} \Rightarrow \mathcal{F}_{1}^{*} \supset \mathcal{F}_{2}^{*}$, and $\mathcal{F}^{* *}=\mathcal{F}$. 
We say that a subset $J$ of $\mathbb{Z}$ has uniform density 1 if for every $0<\lambda<1$ there exists an $N$ such that for every interval $I \subset \mathbb{Z}$ of length $>N$ we have $|J \cap I| \geq \lambda|I|$. We denote by $\mathcal{D}$ the family of subsets of $\mathbb{Z}$ of uniform density 1 . It is also easy to see that $\mathcal{D}$ has the finite intersection property.

Let $\mathcal{F}$ be a family of nonempty subsets of $\mathbb{Z}$ which is closed under finite intersections (i.e. $\mathcal{F}$ is a filter). Following [25] we say that a sequence $\left\{x_{n}: n \in \mathbb{Z}\right\}$ in a topological space $X \mathcal{F}$-converges to a point $x \in X$ if for every neighborhood $V$ of $x$ the set $\left\{n: x_{n} \in V\right\}$ is in $\mathcal{F}$. We denote this by

$$
\mathcal{F}-\lim x_{n}=x .
$$

We have the following characterization of weak mixing for measure preserving systems which explains more clearly its name.

4.4. Theorem. The dynamical system $\mathbf{X}=(X, X, \mu, T)$ is weakly mixing iff for every $A, B \in \mathcal{X}$ we have

$$
\mathcal{D}-\lim \mu\left(T^{-n} A \cap B\right)=\mu(A) \mu(B) .
$$

An analogous characterization of measure theoretical mild mixing is obtained by considering the families of $I P$ and $I P^{*}$ sets. An $I P$-set is any subset of $\mathbb{Z}$ containing a subset of the form $I P\left\{n_{i}\right\}=\left\{n_{i_{1}}+n_{i_{2}}+\cdots+n_{i_{k}}: i_{1}<i_{2}<\cdots<i_{k}\right\}$, for some infinite sequence $\left\{n_{i}\right\}_{i=1}^{\infty}$. We let $\mathcal{J}$ denote the family of $I P$-sets and call the elements of the dual family $\mathcal{J}^{*}, I P^{*}$-sets. Again it is not hard to see that the family of $I P^{*}$-sets is closed under finite intersections. For a proof of the next theorem we refer to [25].

4.5. Theorem. The dynamical system $\mathbf{X}=(X, X, \mu, T)$ is mildly mixing iff for every $A, B \in \mathcal{X}$ we have

$$
\begin{gathered}
\mathcal{J}^{*}-\lim \mu\left(T^{-n} A \cap B\right)=\mu(A) \mu(B) . \\
\text { * }
\end{gathered}
$$

We now turn to the topological category. Let $(X, T)$ be a topological dynamical system. For two non-empty open sets $U, V \subset X$ and a point $x \in X$ set

$$
\begin{gathered}
N(U, V)=\left\{n \in \mathbb{Z}: T^{n} U \cap V \neq \emptyset\right\}, \quad N_{+}(U, V)=N(U, V) \cap \mathbb{Z}_{+} \\
\text {and } \quad N(x, V)=\left\{n \in \mathbb{Z}: T^{n} x \in V\right\} .
\end{gathered}
$$

Notice that sets of the form $N(U, U)$ are symmetric.

We say that $(X, T)$ is topologically transitive (or just transitive) if $N(U, V)$ is nonempty whenever $U, V \subset X$ are two non-empty open sets. Using Baire's category theorem it is easy to see that (for metrizable $X)$ a system $(X, T)$ is topologically transitive iff there exists a dense $G_{\delta}$ subset $X_{0} \subset X$ such that $\overline{\mathcal{O}}_{T}(x)=X$ for every $x \in X_{0}$.

We define the family $\mathcal{F}_{\text {thick }}$ of thick sets to be the collection of sets which contain arbitrary long intervals. The dual family $\mathcal{F}_{\text {synd }}=\mathcal{F}_{\text {thick }}^{*}$ is the collection of syndetic sets - those sets $A \subset \mathbb{Z}$ such that for some positive integer $N$ the intersection of $A$ with every interval of length $N$ is nonempty.

Given a family $\mathcal{F}$ we say that a topological dynamical system $(X, T)$ is $\mathcal{F}$-recurrent if $N(A, A) \in \mathcal{F}$ for every nonempty open set $A \subset X$. We say that a dynamical system is $\mathcal{F}$-transitive if $N(A, B) \in \mathcal{F}$ for every nonempty open sets $A, B \subset X$. The class of 
$\mathcal{F}$-transitive systems is denoted by $\mathcal{E}_{\mathcal{F}}$. E.g. in this notation the class of topologically mixing systems is $\mathcal{E}_{\text {cofinite, }}$, where we call a subset $A \subset \mathbb{Z}$ co-finite when $\mathbb{Z} \backslash A$ is a finite set. We write simply $\mathcal{E}=\mathcal{E}_{\text {infinite }}$ for the class of recurrent transitive dynamical systems. It is not hard to see that when $X$ has no isolated points $(X, T)$ is topologically transitive iff it is recurrent transitive. From this we then deduce that a weakly mixing system is necessarily recurrent transitive.

In a dynamical system $(X, T)$ a point $x \in X$ is a wandering point if there exists an open neighborhood $U$ of $x$ such that the collection $\left\{T^{n} U: n \in \mathbb{Z}\right\}$ is pairwise disjoint.

4.6. Proposition. Let $(X, T)$ be a topologically transitive dynamical system; then the following conditions are equivalent:

1. $(X, T) \in \mathcal{E}_{\text {infinite }}$

2. The recurrent points are dense in $X$.

3. $(X, T)$ has no wandering points.

4. The dynamical system $\left(X_{\infty}, T\right)$, the one point compactification of the integers with translation and a fixed point at infinity, is not a factor of $(X, T)$.

Proof. $1 \Rightarrow 4$ If $\pi: X \rightarrow X_{\infty}$ is a factor map then, clearly $N\left(\pi^{-1}(0), \pi^{-1}(0)\right)=\{0\}$.

$4 \Rightarrow 3$ If $U$ is a nonempty open wandering subset of $X$ then $\left\{T^{j} U: j \in \mathbb{Z}\right\} \cup(X \backslash$ $\left.\bigcup\left\{T^{j} U: j \in \mathbb{Z}\right\}\right)$ is a partition of $X$. It is easy to see that this partition defines a factor map $\pi: X \rightarrow X_{\infty}$.

$3 \Rightarrow 2$ This implication is a consequence of the following:

4.7. Lemma. If the dynamical system $(X, T)$ has no wandering points then the recurrent points are dense in $X$.

Proof. For every $\delta>0$ put

$$
A_{\delta}=\left\{x \in X: \exists j \neq 0, d\left(T^{j} x, x\right)<\delta\right\} .
$$

Clearly $A_{\delta}$ is an open set and we claim that it is dense. In fact given $x \in X$ and $\epsilon>0$ there exists $j \neq 0$ with

$$
T^{j} B_{\epsilon}(x) \cap B_{\epsilon}(x) \neq \emptyset .
$$

If $y$ is a point in this intersection then $d\left(T^{-j} y, y\right)<2 \epsilon$. Thus for $\epsilon<\delta / 2$ we have $y \in A_{\delta}$ and $d(x, y)<\epsilon$. Now by Baire's theorem

$$
A=\bigcap_{k=1}^{\infty} A_{1 / k}
$$

is a dense $G_{\delta}$ subset of $X$ and each point in $A$ is recurrent.

$2 \Rightarrow 1$ Given $U, V$ nonempty open subsets of $X$ and $k \in N(U, V)$ let $U_{0}$ be the nonempty open subset $U_{0}=U \cap T^{-k} V$. Check that $N\left(U_{0}, U_{0}\right)+k \subset N(U, V)$. By assumption $N\left(U_{0}, U_{0}\right)$ is infinite and a fortiori so is $N(U, V)$. This completes the proof of Proposition 4.6.

A well known characterization of the class WM of topologically weakly mixing systems is due to Furstenberg:

4.8. Theorem. $\mathbf{W M}=\mathcal{E}_{\text {thick }}$. 
Following [5] we call the systems in $\mathcal{E}_{\text {synd }}$ topologically ergodic and write TE for this class. This is a rich class as we can see from the following claim from [39. Here MIN is the class of minimal systems and $\mathbf{E}$ the class of $E$-systems; i.e. those transitive dynamical systems $(X, T)$ for which there exists a probability invariant measure with full support.

\subsection{Theorem. MIN, E $\subset$ TE.}

Proof. 1. The claim for MIN is immediate by the well known characterization of minimal systems: $(X, T)$ is minimal iff $N(x, U)$ is syndetic for every $x \in X$ and nonempty open $U \subset X$.

2. Given two non-empty open sets $U, V$ in $X$, choose $k \in \mathbb{Z}$ with $T^{k} U \cap V \neq \emptyset$. Next set $U_{0}=T^{-k} V \cap U$, and observe that $k+N\left(U_{0}, U_{0}\right) \subset N(U, V)$. Thus it is enough to show that $N(U, U)$ is syndetic for every non-empty open $U$. We have to show that $N(U, U)$ meets every thick subset $B \subset \mathbb{Z}$. By Poincaré's recurrence theorem, $N(U, U)$ meets every set of the form $A-A=\{n-m: n, m \in A\}$ with $A$ infinite. It is an easy exercise to show that every thick set $B$ contains some $D^{+}(A)=\left\{a_{n}-a_{m}: n>m\right\}$ for an infinite sequence $A=\left\{a_{n}\right\}$. Thus $\emptyset \neq N(U, U) \cap \pm D^{+}(A) \subset N(U, U) \cap \pm B$. Since $N(U, U)$ is symmetric, this completes the proof.

We recall (see the previous section) that two dynamical systems $(X, T)$ and $(Y, T)$ are disjoint if every closed $T \times T$-invariant subset of $X \times Y$ whose projections on $X$ and $Y$ are full, is necessarily the entire space $X \times Y$. It follows easily that when $(X, T)$ and $(Y, T)$ are disjoint, at least one of them must be minimal. If both $(X, T)$ and $(Y, T)$ are minimal then they are disjoint iff the product system is minimal. We say that $(X, T)$ and $(Y, T)$ are weakly disjoint when the product system $(X \times Y, T \times T)$ is transitive. This is indeed a very weak sense of disjointness as there are systems which are weakly disjoint from themselves. In fact, by definition a dynamical system is topologically weakly mixing iff it is weakly disjoint from itself.

If $\mathbf{P}$ is a class of recurrent transitive dynamical systems we let $\mathbf{P}^{\curlywedge}$ be the class of recurrent transitive dynamical systems which are weakly disjoint from every member of $\mathbf{P}$

$$
\mathbf{P}^{\curlywedge}=\{(X, T): X \times Y \in \mathcal{E} \text { for every }(Y, T) \in \mathcal{P}\} .
$$

We clearly have $\mathbf{P} \subset \mathbf{Q} \Rightarrow \mathbf{P}^{\curlywedge} \supset \mathbf{Q}^{\curlywedge}$ and $\mathbf{P}^{\curlywedge \curlywedge \curlywedge}=\mathbf{P}^{\curlywedge}$.

For the discussion of topologically mildly mixing systems it will be convenient to deal with families of subsets of $\mathbb{Z}_{+}$rather than $\mathbb{Z}$. If $\mathcal{F}$ is such a family then

$$
\mathcal{E}_{\mathcal{F}}=\left\{(X, T): N_{+}(A, B) \in \mathcal{F} \text { for every nonempty open } A, B \subset X\right\} .
$$

Let us call a subset of $\mathbb{Z}_{+}$a $S I P$-set (symmetric $I P$-set), if it contains a subset of the form

$$
S I P\left\{n_{i}\right\}=\left\{n_{\alpha}-n_{\beta}>0: n_{\alpha}, n_{\beta} \in I P\left\{n_{i}\right\} \cup\{0\}\right\},
$$

for an $I P$ sequence $I P\left\{n_{i}\right\} \subset \mathbb{Z}_{+}$. Denote by $\mathcal{S}$ the family of $S I P$ sets. It is not hard to show that

$$
\mathcal{F}_{\text {thick }} \subset \mathcal{S} \subset \mathcal{J}
$$

(see [25]). Hence $\mathcal{F}_{\text {syndetic }} \supset \mathcal{S}^{*} \supset \mathcal{J}^{*}$, hence $\mathcal{E}_{\text {synd }} \supset \mathcal{E}_{\mathcal{S}^{*}} \supset \mathcal{E}_{\mathfrak{J}^{*}}$, and finally

$$
\mathcal{E}_{\text {synd }}^{\curlywedge} \subset \mathcal{E}_{\mathcal{S}^{*}}^{\curlywedge} \subset \mathcal{E}_{\mathrm{J}^{*}}^{\curlywedge} \text {. }
$$


4.10. Definition. A topological dynamical system $(X, T)$ is called topologically mildly mixing if it is in $\mathcal{E}_{\mathcal{S}^{*}}$ and we denote the collection of topologically mildly mixing systems by $\mathbf{M M}=\mathcal{E}_{\mathcal{S}^{*}}$.

4.11. Theorem. A dynamical system is in $\mathcal{E}$ iff it is weakly disjoint from every topologically mildly mixing system:

$$
\mathcal{E}=\mathrm{MM}^{\curlywedge}
$$

And conversely it is topologically mildly mixing iff it is weakly disjoint from every recurrent transitive system:

$$
\mathrm{MM}=\mathcal{E}^{\curlywedge}
$$

Proof. 1. Since $\mathcal{E}_{\mathcal{S}^{*}}$ is nonvacuous (for example every topologically mixing system is in $\left.\mathcal{E}_{\mathcal{S}^{*}}\right)$, it follows that every system in $\mathcal{E}_{\mathcal{S}^{*}}$ is in $\mathcal{E}$.

Conversely, assume that $(\mathrm{X}, \mathrm{T})$ is in $\mathcal{E}$ but $(\mathrm{X}, \mathrm{T}) \notin \mathcal{E}_{\mathcal{S}^{*}}^{\curlywedge}$, and we will arrive at a contradiction. By assumption there exists $(\mathrm{Y}, \mathrm{T}) \in \mathcal{E}_{\mathcal{S}^{*}}$ and a nondense nonempty open invariant subset $W \subset X \times Y$. Then $\pi_{X}(W)=O$ is a nonempty open invariant subset of $X$. By assumption $O$ is dense in $X$. Choose open nonempty sets $U_{0} \subset X$ and $V_{0} \subset Y$ with $U_{0} \times V_{0} \subset W$. By Proposition 4.6 there exists a recurrent point $x_{0}$ in $U_{0} \subset O$. Then there is a sequence $n_{i} \rightarrow \infty$ such that for the $I P$-sequence $\left\{n_{\alpha}\right\}=I P\left\{n_{i}\right\}_{i=1}^{\infty}, I P-\lim T^{n_{\alpha}} x_{0}=x_{0}$ (see [25]). Choose $i_{0}$ such that $T^{n_{\alpha}} x_{0} \in U_{0}$ for $n_{\alpha} \in J=I P\left\{n_{i}\right\}_{i \geq i_{0}}$ and set $D=S I P(J)$. Given $V$ a nonempty open subset of $Y$ we have:

$$
D \cap N\left(V_{0}, V\right) \neq \emptyset .
$$

Thus for some $\alpha, \beta$ and $v_{0} \in V_{0}$,

$$
T^{n_{\alpha}-n_{\beta}}\left(T^{n_{\beta}} x_{0}, v_{0}\right)=\left(T^{n_{\alpha}} x_{0}, T^{n_{\alpha}-n_{\beta}} v_{0}\right) \in\left(U_{0} \times V\right) \cap W .
$$

We conclude that

$$
\left\{x_{0}\right\} \times Y \subset \operatorname{cls} W
$$

The fact that in an $\mathcal{E}$ system the recurrent points are dense together with the observation that $\left\{x_{0}\right\} \times Y \subset \operatorname{cls} W$ for every recurrent point $x_{0} \in O$, imply that $W$ is dense in $X \times Y$, a contradiction.

2. From part 1 of the proof we have $\mathcal{E}=\mathcal{E}_{\mathcal{S}^{*}}^{\curlywedge}$, hence $\mathcal{E}^{\curlywedge}=\mathcal{E}_{\mathcal{S}^{*}}^{\curlywedge} \supset \mathcal{E}_{\mathcal{S}^{*}}$.

Suppose $(\mathrm{X}, \mathrm{T}) \in \mathcal{E}$ but $(\mathrm{X}, \mathrm{T}) \notin \mathcal{E}_{\mathcal{S}^{*}}$, we will show that $(\mathrm{X}, \mathrm{T}) \notin \mathcal{E}^{\curlywedge}$. There exist $U, V \subset X$, nonempty open subsets and an $I P$-set $I=I P\left\{n_{i}\right\}$ for a monotone increasing sequence $\left\{n_{1}<n_{2}<\cdots\right\}$ with

$$
N(U, V) \cap D=\emptyset
$$

where

$$
D=\left\{n_{\alpha}-n_{\beta}: n_{\alpha}, n_{\beta} \in I, n_{\alpha}>n_{\beta}\right\}
$$

If $(\mathrm{X}, \mathrm{T})$ is not topologically weakly mixing then $X \times X \notin \mathcal{E}$ hence $(\mathrm{X}, \mathrm{T}) \notin \mathcal{E}^{\curlywedge}$. So we can assume that $(\mathrm{X}, \mathrm{T})$ is topologically weakly mixing. Now in $X \times X$

$$
N(U \times V, V \times U)=N(U, V) \cap N(V, U)=N(U, V) \cap-N(U, V),
$$

is disjoint from $D \cup-D$, and replacing $X$ by $X \times X$ we can assume that $N(U, V) \cap$ $(D \cup-D)=\emptyset$. In fact, if $X \in \mathcal{E}^{\curlywedge}$ then $X \times Y \in \mathcal{E}$ for every $Y \in \mathcal{E}$, therefore $X \times(X \times Y) \in \mathcal{E}$ and we see that also $X \times X \in \mathcal{E}^{\curlywedge}$. 
By going to a subsequence, we can assume that

$$
\lim _{k \rightarrow \infty} n_{k+1}-\sum_{i=1}^{k} n_{i}=\infty .
$$

in which case the representation of each $n \in I$ as $n=n_{\alpha}=n_{i_{1}}+n_{i_{2}}+\cdots+n_{i_{k}} ; \alpha=$ $\left\{i_{1}<i_{2}<\cdots<i_{k}\right\}$ is unique.

Next let $y_{0} \in\{0,1\}^{\mathbb{Z}}$ be the sequence $y_{0}=\mathbf{1}_{I}$. Let $Y$ be the orbit closure of $y_{0}$ in $\{0,1\}^{\mathbb{Z}}$ under the shift $T$, and let $[1]=\{y \in Y: y(0)=1\}$. Observe that

$$
N\left(y_{0},[1]\right)=I \text {. }
$$

It is easy to check that

$$
I P-\lim T^{n_{\alpha}} y_{0}=y_{0}
$$

Thus the system $(Y, T)$ is topologically transitive with $y_{0}$ a recurrent point; i.e. $(\mathrm{Y}, \mathrm{T}) \in \mathcal{E}$.

We now observe that

$$
N([1],[1])=N\left(y_{0},[1]\right)-N\left(y_{0},[1]\right)=I-I=D \cup-D \cup\{0\} .
$$

If $X \times Y$ is topologically transitive then in particular

$$
\begin{gathered}
N(U \times[1], V \times[1])=N(U, V) \cap N([1],[1])= \\
N(U, V) \cap(D \cup-D \cup\{0\})=\text { infinite set. }
\end{gathered}
$$

But this contradicts our assumption. Thus $X \times Y \notin \mathcal{E}$ and $(\mathrm{X}, \mathrm{T}) \notin \mathcal{E} \curlywedge$. This completes the proof.

We now have the following:

4.12. Corollary. Every topologically mildly mixing system is weakly mixing and topologically ergodic:

$$
\mathrm{MM} \subset \mathbf{W M} \cap \mathrm{TE} .
$$

Proof. We have $\mathcal{E}_{\mathcal{S}^{*}} \subset \mathcal{E}=\mathcal{E}_{\mathcal{S}^{*}}$, hence for every $(\mathrm{X}, \mathrm{T}) \in \mathcal{E}_{\mathcal{S}^{*}}, X \times X \in \mathcal{E}$ i.e. $(\mathrm{X}, \mathrm{T})$ is topologically weakly mixing. And, as we have already observed the inclusion $\mathcal{F}_{\text {syndetic }} \supset \mathcal{S}^{*}$, entails $\mathbf{T E}=\mathcal{E}_{\text {synd }} \supset \mathcal{E}_{\mathcal{S}^{*}}=$ MM.

To complete the analogy with the measure theoretical setup we next define a topological analogue of rigidity. This is just one of several possible definitions of topological rigidity and we refer to [37] for a treatment of these notions.

4.13. Definition. A dynamical system $(X, T)$ is called uniformly rigid if there exists a sequence $n_{k} \nearrow \infty$ such that

$$
\lim _{k \rightarrow \infty} \sup _{x \in X} d\left(T^{n_{k}} x, x\right)=0,
$$

i.e. $\lim _{k \rightarrow \infty} T^{n_{k}}=$ id in the uniform topology on the group of homeomorphism of $H(X)$ of $X$. We denote by $\mathcal{R}$ the collection of topologically transitive uniformly rigid systems.

In [37] the existence of minimal weakly mixing but nonetheless uniformly rigid dynamical systems is demonstrated. However, we have the following: 
4.14. Lemma. A system which is both topologically mildly mixing and uniformly rigid is trivial.

Proof. Let $(\mathrm{X}, \mathrm{T})$ be both topologically mildly mixing and uniformly rigid. Then

$$
\Lambda=\operatorname{cls}\left\{T^{n}: n \in \mathbb{Z}\right\} \subset H(X),
$$

is a Polish monothetic group.

Let $T^{n_{i}}$ be a sequence converging uniformly to id, the identity element of $\Lambda$. For a subsequence we can ensure that $\left\{n_{\alpha}\right\}=\operatorname{IP}\left\{n_{i}\right\}$ is an $I P$-sequence such that $I P-\lim T^{n_{\alpha}}=$ id in $\Lambda$. If $X$ is nontrivial we can now find an open ball $B=B_{\delta}\left(x_{0}\right) \subset X$ with $T B \cap B=\emptyset$. Put $U=B_{\delta / 2}\left(x_{0}\right)$ and $V=T U$; then by assumption $N(U, V)$ is an $S I P^{*}$-set and in particular:

$$
\forall \alpha_{0} \exists \alpha, \beta>\alpha_{0}, n_{\alpha}-n_{\beta} \in N(U, V) .
$$

However, since $I P$ - $\lim T^{n_{\alpha}}=\mathrm{id}$, we also have eventually, $T^{n_{\alpha}-n_{\beta}} U \subset B$; a contradiction.

4.15. Corollary. A topologically mildly mixing system has no nontrivial uniformly rigid factors.

We conclude this section with the following result which shows how these topological and measure theoretical notions are related.

4.16. Theorem. Let $(X, T)$ be a topological dynamical system with the property that there exists an invariant probability measure $\mu$ with full support such that the associated measure preserving dynamical system $(X, X, \mu, T)$ is measure theoretically mildly mixing then $(X, T)$ is topologically mildly mixing.

Proof. Let $(Y, S)$ be any system in $\mathcal{E}$; by Theorem 4.11 it suffices to show that $(X \times$ $Y, T \times S)$ is topologically transitive. Suppose $W \subset X \times Y$ is a closed $T \times S$-invariant set with int $W \neq \emptyset$. Let $U \subset X, V \subset V$ be two nonempty open subsets with $U \times V \subset W$. By transitivity of $(Y, S)$ there exits a transitive recurrent point $y_{0} \in V$. By theorems of Glimm and Effros [42], [21], and Katznelson and Weiss [56] (see also Weiss [82]), there exists a (possibly infinite) invariant ergodic measure $\nu$ on $Y$ with $\nu(V)>0$.

Let $\mu$ be the probability invariant measure of full support on $X$ with respect to which $(X, X, \mu, T)$ is measure theoretically mildly mixing. Then by [27] the measure $\mu \times \nu$ is ergodic. Since $\mu \times \nu(W) \geq \mu \times \nu(U \times V)>0$ we conclude that $\mu \times \nu\left(W^{c}\right)=0$ which clearly implies $W=X \times Y$.

We note that the definition of topological mild mixing and the results described above concerning this notion are new. However independently of our work Huang and Ye in a recent work also define a similar notion and give it a comprehensive and systematic treatment, [4]. The first named author would like to thank E. Akin for instructive conversations on this subject.

Regarding the classes WM and TE let us mention the following result from [85].

\subsection{Theorem.}

$$
\mathrm{TE}=\mathrm{WM}^{\curlywedge} \text {. }
$$

For more on these topics we refer to [25], 3], 85, [5], 48] and [49]. 


\section{Distal Systems: TOPOlOGiCAL VS. MeAsure}

As noted above the Kronecker or minimal equicontinuous dynamical systems can be considered as the most elementary type of systems. What is then the next stage? The clue in the topological case, which chronologically came first, is to be found in the notion of distality. A topological system $(X, T)$ is called distal if

$$
\inf _{n \in \mathbb{Z}} d\left(T^{n} x, T^{n} x^{\prime}\right)>0
$$

for every $x \neq x^{\prime}$ in $X$. It is easy to see that this property does not depend on the choice of a metric. And, of course, every equicontinuous system is distal. Is the converse true? Are these notions one and the same? The dynamical system given on the unit $\operatorname{disc} D=\{z \in \mathbb{C}:|z| \leq 1\}$ by the formula $T z=z \exp (2 \pi i|z|)$ is a counter example, it is distal but not equicontinuous. However it is not minimal. H. Furstenberg in 1963 noted that skew products over an equicontinuous basis with compact group translations as fiber maps are always distal, often minimal, but rarely equicontinuous, [22. A typical example is the homeomorphism of the two torus $\mathbb{T}^{2}=\mathbb{R}^{2} / \mathbb{Z}^{2}$ given by $T(x, y)=(x+\alpha, y+x)$ where $\alpha \in \mathbb{R} / Z$ is irrational. Independently and at about the same time, it was shown by L. Auslander, L. Green and F. Hahn that minimal nilflows are distal but not equicontinuous, 6]. These examples led Furstenberg to his path breaking structure theorem, $[22$.

Given a homomorphism $\pi:(X, T) \rightarrow(Y, T)$ let $R_{\pi}=\left\{\left(x, x^{\prime}\right): \pi(x)=\pi\left(x^{\prime}\right)\right\}$. We say that the homomorphism $\pi$ is an isometric extension if there exists a continuous function $d: R_{\pi} \rightarrow \mathbb{R}$ such that for each $y \in Y$ the restriction of $d$ to $\pi^{-1}(y) \times \pi^{-1}(y)$ is a metric and for every $x, x^{\prime} \in \pi^{-1}(y)$ we have $d\left(T x, T x^{\prime}\right)=d\left(x, x^{\prime}\right)$.

If $K$ is a compact subgroup of $\operatorname{Aut}(X, T)$ (the group of homeomorphisms of $X$ commuting with $T$, endowed with the topology of uniform convergence) then the map $x \mapsto K x$ defines a factor map $(X, T) \stackrel{\pi}{\rightarrow}(Y, T)$ with $Y=X / K$ and $R_{\pi}=$ $\{(x, k x): x \in X, k \in K\}$. Such an extension is called a group extension. It turns out, although this is not so easy to see, that when $(X, T)$ is minimal then $\pi:(X, T) \rightarrow(Y, T)$ is an isometric extension iff there exists a commutative diagram:

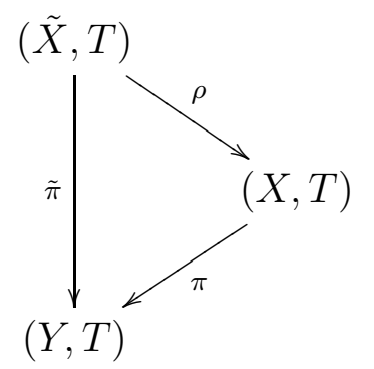

where $(\tilde{X}, T)$ is minimal and $(\tilde{X}, T) \stackrel{\tilde{\pi}}{\rightarrow}(X, T)$ is a group extension with some compact group $K \subset \operatorname{Aut}(\tilde{X}, T)$ and the map $\rho$ is the quotient map from $\tilde{X}$ onto $X$ defined by a closed subgroup $H$ of $K$. Thus $Y=\tilde{X} / K$ and $X=\tilde{X} / H$ and we can think of $\pi$ as a homogeneous space extension with fiber $K / H$.

We say that a (metrizable) minimal system $(X, T)$ is an $I$ system if there is a (countable) ordinal $\eta$ and a family of systems $\left\{\left(X_{\theta}, x_{\theta}\right)\right\}_{\theta \leq \eta}$ such that (i) $X_{0}$ is the trivial system, (ii) for every $\theta<\eta$ there exists an isometric homomorphism 
$\phi_{\theta}: X_{\theta+1} \rightarrow X_{\theta}$, (iii) for a limit ordinal $\lambda \leq \eta$ the system $X_{\lambda}$ is the inverse limit of the systems $\left\{X_{\theta}\right\}_{\theta<\lambda}$ (i.e. $X_{\lambda}=\bigvee_{\theta<\lambda}\left(X_{\theta}, x_{\theta}\right)$ ), and (iv) $X_{\eta}=X$.

5.1. Theorem (Furstenberg's structure theorem). A minimal system is distal iff it is an I-system.

W. Parry in his 1967 paper [69] suggested an intrinsic definition of measure distality. He defines in this paper a property of measure dynamical systems, called "admitting a separating sieve", which imitates the intrinsic definition of topological distality.

5.2. Definition. Let $\mathbf{X}$ be an ergodic dynamical system. A sequence $A_{1} \supset A_{2} \supset \ldots$ of sets in $X$ with $\mu\left(A_{n}\right)>0$ and $\mu\left(A_{n}\right) \rightarrow 0$, is called a separating sieve if there exists a subset $X_{0} \subset X$ with $\mu\left(X_{0}\right)=1$ such that for every $x, x^{\prime} \in X_{0}$ the condition "for every $n \in \mathbb{N}$ there exists $k \in \mathbb{Z}$ with $T^{k} x, T^{k} x^{\prime} \in A_{n}$ " implies $x=x^{\prime}$, or in symbols:

$$
\bigcap_{n=1}^{\infty}\left(\bigcup_{k \in \mathbb{Z}} T^{k}\left(A_{n} \times A_{n}\right)\right) \cap\left(X_{0} \times X_{0}\right) \subset \Delta .
$$

We say that the ergodic system $\mathbf{X}$ is measure distal if either $\mathbf{X}$ is finite or there exists a separating sieve.

Parry showed that every measure dynamical system admitting a separating sieve has zero entropy and that any $T$-invariant measure on a minimal topologically distal system gives rise to a measure dynamical system admitting a separating sieve.

If $\mathbf{X}=(X, X, \mu, T)$ is an ergodic dynamical system and $K \subset \operatorname{Aut}(\mathbf{X})$ is a compact subgroup (where Aut $(\mathbf{X})$ is endowed with the weak topology) then the system $\mathbf{Y}=\mathbf{X} / K$ is well defined and we say that the extension $\pi: \mathbf{X} \rightarrow \mathbf{Y}$ is a group extension. Using (5) we can define the notion of isometric extension or homogeneous extension in the measure category. We will say that an ergodic system admits a Furstenberg tower if it is obtained as a (necessarily countable) transfinite tower of measure isometric extensions. In 1976 in two outstanding papers [87, [88 R. Zimmer developed the theory of distal systems (for a general locally compact acting group). He showed that, as in the topologically distal case, systems admitting Parry's separating sieve are exactly those systems which admit Furstenberg towers.

5.3. Theorem. An ergodic dynamical system is measure distal iff it admits a Furstenberg tower.

In 64] E. Lindenstrauss shows that every ergodic measure distal $\mathbb{Z}$-system can be represented as a minimal topologically distal system. For the exact result see Theorem 13.4 below. 


\section{Furstenberg-Zimmer structure theorem vs. its topological PI VERSION}

Zimmer's theorem for distal systems leads directly to a structure theorem for the general ergodic system. Independently, and at about the same time, Furstenberg proved the same theorem, [24, [25]. He used it as the main tool for his proof of Szemerédi's theorem on arithmetical progressions. Recall that an extension $\pi:(X, X, \mu, T) \rightarrow(Y, y, \nu, T)$ is a weakly mixing extension if the relative product system $\mathbf{X} \underset{\mathbf{Y}}{\times} \mathbf{X}$ is ergodic. (The system $\underset{\mathbf{X}}{\mathbf{X}} \underset{\mathbf{X}}{\mathbf{X}}$ is defined by the $T \times T$ invariant measure

$$
\mu \underset{\nu}{\times} \mu=\int_{Y} \mu_{y} \times \mu_{y} d \nu(y),
$$

on $X \times X$, where $\mu=\int_{Y} \mu_{y} d \nu(y)$ is the disintegration of $\mu$ over $\nu$.)

6.1. Theorem (The Furstenberg-Zimmer structure theorem). Let $\mathbf{X}$ be an ergodic dynamical system.

1. There exists a maximal distal factor $\phi: \mathbf{X} \rightarrow \mathbf{Z}$ with $\phi$ is a weakly mixing extension.

2. This factorization is unique.

*

Is there a general structure theorem for minimal topological systems? Here, for the first time, we see a strong divergence between the measure and the topological theories. The culpability for this divergence is to be found in the notions of proximality and proximal extension, which arise naturally in the topological theory but do not appear at all in the measure theoretical context. In building towers for minimal systems we have to use two building blocks of extremely different nature (isometric and proximal) rather than one (isometric) in the measure category. A pair of points $\left(x, x^{\prime}\right) \in X \times X$ is called proximal if it is not distal, i.e. if $\inf _{n \in \mathbb{Z}} d\left(T^{n} x, T^{n} x^{\prime}\right)=0$. An extension $\pi:(X, T) \rightarrow(Y, T)$ is called proximal if every pair in $R_{\pi}$ is proximal. The next theorem was developed gradually by several authors (Veech, GlasnerEllis-Shapiro, and McMahon, [79], 29], 65], [80]). We need first to introduce some definitions. We say that a minimal dynamical system $(X, T)$ is strictly $\mathbf{P I}$ (proximal isometric) if it admits a tower consisting of proximal and isometric extensions. It is called a PI system if there is a strictly PI minimal system $(\tilde{X}, T)$ and a proximal extension $\theta: \tilde{X} \rightarrow X$. An extension $\pi: X \rightarrow Y$ is a RIC extension (relatively incontractible) if for every $n \in \mathbb{N}$ and every $y \in Y$ the set of almost periodic points in $X_{y}^{n}=\pi^{-1}(y) \times \pi^{-1}(y) \times \cdots \times \pi^{-1}(y)$ ( $n$ times) is dense. (A point is called almost periodic if its orbit closure is minimal.) It can be shown that a every isometric (and more generally, distal) extension is RIC. Also every RIC extension is open. Finally a homomorphism $\pi: X \rightarrow Y$ is called topologically weakly mixing if the dynamical system $\left(R_{\pi}, T \times T\right)$ is topologically transitive.

The philosophy in the next theorem is to regard proximal extensions as 'negligible' and then the claim is, roughly (i.e. up to proximal extensions), that every minimal system is a weakly mixing extension of its maximal PI factor. 
6.2. Theorem (Structure theorem for minimal systems). Given a metric minimal system $(X, T)$, there exists a countable ordinal $\eta$ and a canonically defined commutative diagram (the canonical PI-Tower)

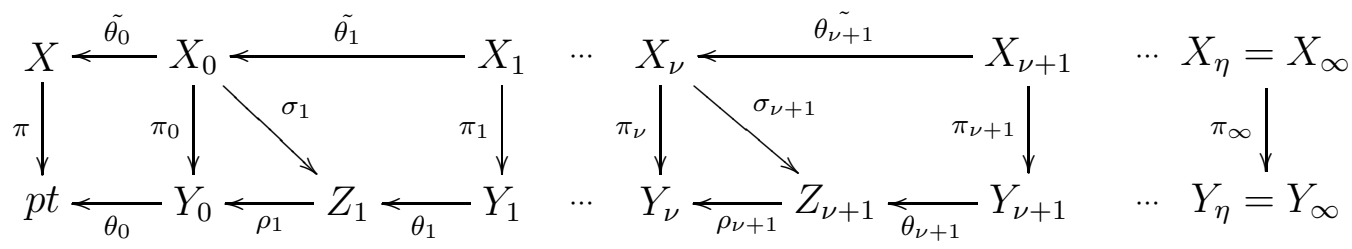

where for each $\nu \leq \eta, \pi_{\nu}$ is $R I C, \rho_{\nu}$ is isometric, $\theta_{\nu}, \tilde{\theta}_{\nu}$ are proximal extensions and $\pi_{\infty}$ is RIC and topologically weakly mixing extension. For a limit ordinal $\nu, X_{\nu}, Y_{\nu}, \pi_{\nu}$ etc. are the inverse limits (or joins) of $X_{\iota}, Y_{\iota}, \pi_{\iota}$ etc. for $\iota<\nu$. Thus $X_{\infty}$ is a proximal extension of $X$ and a RIC topologically weakly mixing extension of the strictly PIsystem $Y_{\infty}$. The homomorphism $\pi_{\infty}$ is an isomorphism (so that $X_{\infty}=Y_{\infty}$ ) iff $X$ is a PI-system.

We refer to [33] for a review on structure theory in topological dynamics.

\section{ENTROPY: MEASURE AND TOPOLOGICAL}

7.1. The classical variational principle. For the definitions and the classical results concerning entropy theory we refer to [46], Section 3.7 for measure theory entropy and Section 4.4 for metric and topological entropy. The variational principle asserts that for a topological $\mathbb{Z}$-dynamical system $(X, T)$ the topological entropy equals the supremum of the measure entropies computed over all the invariant probability measures on $X$. It was already conjectured in the original paper of Adler, Konheim and McAndrew [2] where topological entropy was introduced; and then, after many stages (mainly by Goodwyn, Bowen and Dinaburg; see for example [17]) matured into a theorem in Goodman's paper [43].

7.1. Theorem (The variational principle). Let $(X, T)$ be a topological dynamical system, then

$$
h_{\text {top }}(X, T)=\sup \left\{h_{\mu}: \mu \in M_{T}(X)\right\}=\sup \left\{h_{\mu}: \mu \in M_{T}^{\text {erg }}(X)\right\} .
$$

This classical theorem has had a tremendous influence on the theory of dynamical systems and a vast amount of literature ensued, which we will not try to trace here (see [46, Theorem 4.4.4]). Instead we would like to present a more recent development.

7.2. Entropy pairs and UPE systems. As we have noted in the introduction, the theories of measurable dynamics (ergodic theory) and topological dynamics exhibit a remarkable parallelism. Usually one translates 'ergodicity' as 'topological transitivity', 'weak mixing' as 'topological weak mixing', 'mixing' as 'topological mixing' and 'measure distal' as 'topologically distal'. One often obtains this way parallel theorems in both theories, though the methods of proof may be very different.

What is then the topological analogue of being a K-system? In 8 and 9 F. Blanchard introduced a notion of 'topological $K$ ' for $\mathbb{Z}$-systems which he called UPE (uniformly positive entropy). This is defined as follows: a topological dynamical 
system $(X, T)$ is called a UPE system if every open cover of $X$ by two non-dense open sets $U$ and $V$ has positive topological entropy. A local version of this definition led to the concept of an entropy pair. A pair $\left(x, x^{\prime}\right) \in X \times X, x \neq x^{\prime}$ is an entropy pair if for every open cover $\mathcal{U}=\{U, V\}$ of $X$, with $x \in \operatorname{int}\left(U^{c}\right)$ and $x^{\prime} \in \operatorname{int}\left(V^{c}\right)$, the topological entropy $h(\mathcal{U})$ is positive. The set of entropy pairs is denoted by $E_{X}=E_{(X, T)}$ and it follows that the system $(X, T)$ is UPE iff $E_{X}=(X \times X) \backslash \Delta$. In general $E^{*}=E_{X} \cup \Delta$ is a $T \times T$-invariant closed symmetric and reflexive relation. Is it also transitive? When the answer to this question is affirmative then the quotient system $X / E_{X}^{*}$ is the topological analogue of the Pinsker factor. Unfortunately this need not always be true even when $(X, T)$ is a minimal system (see [41] for a counter example).

The following theorem was proved in Glasner and Weiss [40].

7.2. Theorem. If the compact system $(X, T)$ supports an invariant measure $\mu$ for which the corresponding measure theoretical system $(X, X, \mu, T)$ is a K-system, then $(X, T)$ is UPE.

Applying this theorem together with the Jewett-Krieger theorem it is now possible to obtain a great variety of strictly ergodic UPE systems.

Given a $T$-invariant probability measure $\mu$ on $X$, a pair $\left(x, x^{\prime}\right) \in X \times X, x \neq$ $x^{\prime}$ is called a $\mu$-entropy pair if for every Borel partition $\alpha=\left\{Q, Q^{c}\right\}$ of $X$ with $x \in \operatorname{int}(Q)$ and $x^{\prime} \in \operatorname{int}\left(Q^{c}\right)$ the measure entropy $h_{\mu}(\alpha)$ is positive. This definition was introduced by Blanchard, Host, Maass, Martínez and Rudolph in [12] as a local generalization of Theorem[7.2, It was shown in [12] that for every invariant probability measure $\mu$ the set $E_{\mu}$ of $\mu$-entropy pairs is contained in $E_{X}$.

\subsection{Theorem. Every measure entropy pair is a topological entropy pair.}

As in [40] the main issue here is to understand the, sometimes intricate, relation between the combinatorial entropy $h_{c}(\mathcal{U})$ of a cover $\mathcal{U}$ and the measure theoretical entropy $h_{\mu}(\gamma)$ of a measurable partition $\gamma$ subordinate to $\mathcal{U}$.

7.4. Proposition. Let $\mathbf{X}=(X, X, \mu, T)$ be a measure dynamical system. Suppose $\mathcal{U}=\{U, V\}$ is a measurable cover such that every measurable two-set partition $\gamma=$ $\left\{H, H^{c}\right\}$ which (as a cover) is finer than $\mathcal{U}$ satisfies $h_{\mu}(\gamma)>0$; then $h_{c}(\mathcal{U})>0$.

Since for a $K$-measure $\mu$ clearly every pair of distinct points is in $E_{\mu}$, Theorem 7.2 follows from Theorem 7.3 . It was shown in 12 that when $(X, T)$ is uniquely ergodic the converse of Theorem 7.3 is also true: $E_{X}=E_{\mu}$ for the unique invariant measure $\mu$ on $X$.

7.3. A measure attaining the topological entropy of an open cover. In order to gain a better understanding of the relationship between measure entropy pairs and topological entropy pairs one direction of a variational principle for open covers (Theorem 7.5 below) was proved in Blanchard, Glasner and Host [10. Two applications of this principle were given in 10; (i) the construction, for a general system $(X, T)$, of a measure $\mu \in M_{T}(X)$ with $E_{X}=E_{\mu}$, and (ii) the proof that under a homomorphism $\pi:(X, \mu, T) \rightarrow(Y, \nu, T)$ every entropy pair in $E_{\nu}$ is the image of an entropy pair in $E_{\mu}$. 
We now proceed with the statement and proof of this theorem which is of independent interest. The other direction of this variational principle will be proved in the following subsection.

7.5. Theorem. Let $(X, T)$ be a topological dynamical system, and $\mathcal{U}$ an open cover of $X$, then there exists a measure $\mu \in M_{T}(X)$ such that $h_{\mu}(\alpha) \geq h_{\mathrm{top}}(\mathcal{U})$ for all Borel partitions a finer than $\mathcal{U}$.

A crucial element of the proof of the variational principle is a combinatorial lemma which we present next. We let $\phi:[0,1] \rightarrow \mathbb{R}$ denote the function

$$
\phi(x)=-t \log t \quad \text { for } 0<t \leq 1 ; \phi(0)=0 .
$$

Let $\mathfrak{L}=\{1,2, \ldots, \ell\}$ be a finite set, called the alphabet; sequences $\omega=\omega_{1} \ldots \omega_{n} \in$ $\mathfrak{L}^{n}$, for $n \geq 1$, are called words of length $n$ on the alphabet $\mathfrak{L}$. Let $n$ and $k$ be two integers with $1 \leq k \leq n$.

For every word $\omega$ of length $n$ and every word $\theta$ of length $k$ on the same alphabet, we denote by $p(\theta \mid \omega)$ the frequency of appearances of $\theta$ in $\omega$, i.e.

$$
p(\theta \mid \omega)=\frac{1}{n-k+1} \operatorname{card}\left\{i: 1 \leq i \leq n-k+1, \omega_{i} \omega_{i+1} \ldots \omega_{i+k-1}=\theta_{1} \theta_{2} \ldots \theta_{k}\right\} .
$$

For every word $\omega$ of length $n$ on the alphabet $\mathfrak{L}$, we let

$$
H_{k}(\omega)=\sum_{\theta \in \mathfrak{L}^{k}} \phi(p(\theta \mid \omega))
$$

7.6. Lemma. For every $h>0, \epsilon>0$, every integer $k \geq 1$ and every sufficiently large integer $n$,

$$
\text { card }\left\{\omega \in \mathfrak{L}^{n}: H_{k}(\omega) \leq k h\right\} \leq \exp (n(h+\epsilon))
$$

Remark. It is equally true that, if $h \leq \log (\operatorname{card} \mathfrak{L})$, for sufficiently large $n$,

$$
\text { card }\left\{\omega \in \mathfrak{L}^{n}: H_{k}(\omega) \leq k h\right\} \geq \exp (n(h-\epsilon)) .
$$

We do not prove this inequality here, since we have no use for it in the sequel.

Proof. The case $k=1$.

We have

$$
\operatorname{card}\left\{\omega \in \mathfrak{L}^{n}: H_{1}(\omega) \leq h\right\}=\sum_{q \in K} \frac{n !}{q_{1} ! \ldots q_{\ell} !}
$$

where $K$ is the set of $q=\left(q_{1}, \ldots, q_{\ell}\right) \in \mathbb{N}^{\ell}$ such that

$$
\sum_{i=1}^{\ell} q_{i}=n \text { and } \sum_{i=1}^{\ell} \phi\left(\frac{q_{i}}{n}\right) \leq h .
$$

By Stirling's formula, there exist two universal constants $c$ and $c^{\prime}$ such that

$$
c\left(\frac{m}{e}\right)^{m} \sqrt{m} \leq m ! \leq c^{\prime}\left(\frac{m}{e}\right)^{m} \sqrt{m}
$$

for every $m>0$. From this we deduce the existence of a constant $C(\ell)$ such that for every $q \in K$,

$$
\frac{n !}{q_{1} ! \ldots q_{\ell} !} \leq C(\ell) \exp \left(n \sum_{i=1}^{\ell} \phi\left(\frac{q_{i}}{n}\right)\right) \leq C(\ell) \exp (n h)
$$


Now the sum (2) contains at most $(n+1)^{\ell}$ terms; so that we have

$$
\text { card }\left\{\omega \in \mathfrak{L}^{n}: H_{1}(\omega) \leq h\right\} \leq(n+1)^{\ell} C(\ell) \exp (n h) \leq \exp (n(h+\epsilon))
$$

for all sufficiently large $n$, as was to be proved.

The case $k>1$.

For every word $\omega$ of length $n \geq 2 k$ on the alphabet $\mathfrak{L}$, and for $0 \leq j<k$, we let $n_{j}$ be the integral part of $\frac{n-j}{k}$, and $\omega^{(j)}$ the word

$$
\left(\omega_{j+1} \ldots \omega_{j+k}\right)\left(\omega_{j+k+1} \ldots \omega_{j+2 k}\right) \ldots\left(\omega_{j+\left(n_{j}-1\right) k+1} \ldots \omega_{j+n_{j} k}\right)
$$

of length $n_{j}$ on the alphabet $B=\mathfrak{L}^{k}$.

Let now $\theta$ be a word of length $k$ on the alphabet $\mathfrak{L}$; we also consider $\theta$ as an element of $B$. One easily verifies that, for every word $\omega$ of length $n$ on the alphabet $\mathfrak{L}$,

$$
\left|p(\theta \mid \omega)-\frac{1}{k} \sum_{j=0}^{k-1} p\left(\theta \mid \omega^{(j)}\right)\right| \leq \frac{k}{n-2 k+1} .
$$

The function $\phi$ being uniformly continuous, we see that for sufficiently large $n$, and for every word $\omega$ of length $n$ on $\mathfrak{L}$,

$$
\sum_{\theta \in B}\left|\phi(p(\theta \mid \omega))-\phi\left(\frac{1}{k} \sum_{j=0}^{k-1} p\left(\theta \mid \omega^{(j)}\right)\right)\right|<\frac{\epsilon}{2}
$$

and by convexity of $\phi$,

$$
\frac{1}{k} \sum_{j=0}^{k-1} H_{1}\left(\omega^{(j)}\right)=\frac{1}{k} \sum_{j=0}^{k-1} \sum_{\theta \in B} \phi\left(p\left(\theta \mid \omega^{(j)}\right)\right) \leq \frac{\epsilon}{2}+\sum_{\theta \in \mathfrak{L}^{k}} \phi(p(\theta \mid \omega))=\frac{\epsilon}{2}+H_{k}(\omega) .
$$

Thus, if $H_{k}(\omega) \leq k h$, there exists a $j$ such that $H_{1}\left(\omega^{(j)}\right) \leq \frac{\epsilon}{2}+k h$.

Now, given $j$ and a word $u$ of length $n_{j}$ on the alphabet $B$, there exist $\ell^{n-n_{j} k} \leq \ell^{2 k-2}$ words $\omega$ of length $n$ on $\mathfrak{L}$ such that $\omega^{(j)}=u$. Thus for sufficiently large $n$, by the first part of the proof,

$$
\begin{aligned}
\operatorname{card}\left\{\omega \in \mathfrak{L}^{n}: H_{k}(\omega) \leq k h\right\} & \leq \ell^{2 k-2} \sum_{j=0}^{k-1} \operatorname{card}\left\{u \in B^{n_{j}}: H_{1}(u) \leq \frac{\epsilon}{2}+k h\right\} \\
& \leq \ell^{2 k-2} \sum_{j=0}^{k-1} \exp \left(n_{j}(\epsilon+k h)\right) \\
& \leq \ell^{2 k-2} k \exp \left(n\left(\frac{\epsilon}{k}+h\right)\right) \leq \exp (n(h+\epsilon)) .
\end{aligned}
$$

Let $(X, T)$ be a compact dynamical system. As usual we denote by $M_{T}(X)$ the set of $T$-invariant probability measures on $X$, and by $M_{T}^{\mathrm{erg}}(X)$ the subset of ergodic measures.

We say that a partition $\alpha$ is finer than a cover $\mathcal{U}$ when every atom of $\alpha$ is contained in an element of $\mathcal{U}$. If $\alpha=\left\{A_{1}, \ldots, A_{\ell}\right\}$ is a partition of $X, x \in X$ and $N \in \mathbb{N}$, we write $\omega(\alpha, N, x)$ for the word of length $N$ on the alphabet $\mathfrak{L}=\{1, \ldots, \ell\}$ defined by

$$
\omega(\alpha, N, x)_{n}=i \quad \text { if } \quad T^{n-1} x \in A_{i}, \quad 1 \leq n \leq N .
$$


7.7. Lemma. Let $\mathcal{U}$ be a cover of $X, h=h_{\text {top }}(\mathcal{U}), K \geq 1$ an integer, and $\left\{\alpha_{l}: 1 \leq\right.$ $l \leq K\}$ a finite sequence of partitions of $X$, all finer than $\mathcal{U}$. For every $\epsilon>0$ and sufficiently large $N$, there exists an $x \in X$ such that

$$
H_{k}\left(\omega\left(\alpha_{l}, N, x\right)\right) \geq k(h-\epsilon) \text { for every } k, l \text { with } 1 \leq k, l \leq K .
$$

Proof. One can assume that all the partitions $\alpha_{l}$ have the same number of elements $\ell$ and we let $\mathfrak{L}=\{1, \ldots, \ell\}$. For $1 \leq k \leq K$ and $N \geq K$, denote

$$
\Omega(N, k)=\left\{\omega \in \mathfrak{L}^{N}: H_{k}(\omega)<k(h-\epsilon)\right\} .
$$

By Lemma 7.3, for sufficiently large $N$

$$
\operatorname{card}(\Omega(N, k)) \leq \exp (N(h-\epsilon / 2)) \text { for all } k \leq K .
$$

Let us choose such an $N$ which moreover satisfies $K^{2}<\exp (N \epsilon / 2)$. For $1 \leq k, l \leq K$, let

$$
Z(k, l)=\left\{x \in X: \omega\left(\alpha_{l}, N, x\right) \in \Omega(N, k)\right\} .
$$

The set $Z(k, l)$ is the union of card $(\Omega(N, k))$ elements of $\left(\alpha_{l}\right)_{0}^{N-1}$. Now this partition is finer than the cover $\mathcal{U}_{0}^{N-1}$, hence $Z(k, l)$ is covered by

$$
\operatorname{card}(\Omega(N, k)) \leq \exp (N(h-\epsilon / 2))
$$

elements of $\mathcal{U}_{0}^{N-1}$. Finally,

$$
\bigcup_{1 \leq k, l \leq K} Z(k, l)
$$

is covered by $K^{2} \exp (N(h-\epsilon / 2))<\exp (N h)$ elements of $\mathcal{U}_{0}^{N-1}$. As every subcover of $\mathcal{U}_{0}^{N-1}$ has at least $\exp (N h)$ elements,

$$
\bigcup_{1 \leq k, l \leq K} Z(k, l) \neq X
$$

This completes the proof of the lemma.

Proof of theorem 7.5. Let $\mathcal{U}=\left\{U_{1}, \ldots, U_{\ell}\right\}$ be an open cover of $X$. It is clearly sufficient to consider Borel partitions $\alpha$ of $X$ of the form

$$
\alpha=\left\{A_{1}, \ldots, A_{\ell}\right\} \text { with } A_{i} \subset U_{i} \text { for every } i .
$$

Step 1: Assume first that $X$ is 0 -dimensional.

The family of partitions finer than $\mathcal{U}$, consisting of clopen sets and satisfying (3) is countable; let $\left\{\alpha_{l}: l \geq 1\right\}$ be an enumeration of this family. According to the previous lemma, there exists a sequence of integers $N_{K}$ tending to $+\infty$ and a sequence $x_{K}$ of elements of $X$ such that:

$$
H_{k}\left(\omega\left(\alpha_{l}, N_{K}, x_{K}\right)\right) \geq k\left(h-\frac{1}{K}\right) \text { for every } 1 \leq k, l \leq K .
$$

Write

$$
\mu_{K}=\frac{1}{N_{K}} \sum_{i=0}^{N_{K}-1} \delta_{T^{i} x_{K}} .
$$

Replacing the sequence $\mu_{K}$ by a subsequence (this means replacing the sequence $N_{K}$ by a subsequence, and the sequence $x_{K}$ by the corresponding subsequence preserving the property (4) ), one can assume that the sequence of measures $\mu_{K}$ converges weak* 
to a probability measure $\mu$. This measure $\mu$ is clearly $T$-invariant. Fix $k, l \geq 1$, and let $F$ be an atom of the partition $\left(\alpha_{l}\right)_{0}^{k-1}$, with name $\theta \in\{1, \ldots, \ell\}^{k}$. For every $K$ one has

Now as $F$ is clopen,

$$
\left|\mu_{K}(F)-p\left(\theta \mid \omega\left(\alpha_{l}, N_{K}, x_{K}\right)\right)\right| \leq \frac{2 k}{N_{K}}
$$

$$
\begin{aligned}
\mu(F) & =\lim _{K \rightarrow \infty} \mu_{K}(F)=\lim _{K \rightarrow \infty} p\left(\theta \mid \omega\left(\alpha_{l}, N_{K}, x_{K}\right)\right) \text { hence } \\
\phi(\mu(F)) & =\lim _{K \rightarrow \infty} \phi\left(p\left(\theta \mid \omega\left(\alpha_{l}, N_{K}, x_{K}\right)\right)\right)
\end{aligned}
$$

and, summing over $\theta \in\{1, \ldots, \ell\}^{k}$, one gets

$$
H_{\mu}\left(\left(\alpha_{l}\right)_{0}^{k-1}\right)=\lim _{K \rightarrow \infty} H_{k}\left(\omega\left(\alpha_{l}, N_{K}, x_{K}\right)\right) \geq k h .
$$

Finally, by sending $k$ to infinity one obtains $h_{\mu}\left(\alpha_{l}\right) \geq h$.

Now, as $X$ is 0 -dimensional, the family of partitions $\left\{\alpha_{l}\right\}$ is dense in the collection of Borel partitions of $X$ satisfying (3), with respect to the distance associated with $L^{1}(\mu)$. Thus, $h_{\mu}(\alpha) \geq h$ for every partition of this kind.

Step 2: The general case.

Let us recall a well known fact: there exists a topological system $(Y, T)$, where $Y$ is 0-dimensional, and a continuous surjective map $\pi: Y \rightarrow X$ with $\pi \circ T=T \circ \pi$.

(Proof : as $X$ is a compact metric space, it is easy to construct a Cantor set $K$ and a continuous surjective $f: K \rightarrow X$. Put

$$
Y=\left\{y \in K^{\mathbb{Z}}: f\left(y_{n+1}\right)=T f\left(y_{n}\right) \text { for every } n \in \mathbb{Z}\right\}
$$

and let $\pi: Y \rightarrow X$ be defined by $\pi(y)=f\left(y_{0}\right)$.

$Y$ is a closed subset of $K^{\mathbb{Z}}$ - where the latter is equipped with the product topology - and is invariant under the shift $T$ on $K^{\mathbb{Z}}$. It is easy to check that $\pi$ satisfies the required conditions.)

Let $\mathcal{V}=\pi^{-1}(\mathcal{U})=\left\{\pi^{-1}\left(U_{1}\right), \ldots, \pi^{-1}\left(U_{d}\right)\right\}$ be the preimage of $\mathcal{U}$ under $\pi$; one has $h_{\text {top }}(\mathcal{V})=h_{\text {top }}(\mathcal{U})=h$. By the above remark, there exists $\nu \in M(Y, T)$ such that $h_{\nu}(\mathcal{Q}) \geq h$ for every Borel partition $\mathcal{Q}$ of $Y$ finer than $\mathcal{V}$. Let $\mu=\nu \circ \pi^{-1}$ the measure which is the image of $\nu$ under $\pi$. One has $\mu \in M_{T}(X)$ and, for every Borel partition $\alpha$ of $X$ finer than $\mathcal{U}, \pi^{-1}(\alpha)$ is a Borel partition of $Y$ which is finer than $\mathcal{V}$ with

$$
h_{\mu}(\alpha)=h_{\nu}\left(\pi^{-1}(\alpha)\right) \geq h .
$$

This completes the proof of the theorem.

7.8. Corollary. Let $(X, T)$ be a topological system, $\mathcal{U}$ an open cover of $X$ and $\alpha$ a Borel partition finer than $\mathcal{U}$, then, there exists a $T$-invariant ergodic measure $\mu$ on $X$ such that $h_{\mu}(\alpha) \geq h_{\mathrm{top}}(\mathcal{U})$.

Proof. By Theorem [7.5 there exists $\mu \in M_{T}(X)$ with $h_{\mu}(\alpha) \geq h_{\text {top }}(\mathcal{U})$; let $\mu=$ $\int_{\omega} \mu_{\omega} d m(\omega)$ be its ergodic decomposition. The corollary follows from the formula

$$
\int h_{\mu_{\omega}}(\alpha) d m(\omega)=h_{\mu}(\alpha)
$$


7.4. The variational principle for open covers. Given an open cover $\mathcal{U}$ of the dynamical system $(X, T)$, the results of the previous subsection imply the inequality

$$
\sup _{\mu \in M_{T}(X)} \inf _{\alpha \succ U} h_{\mu}(\alpha) \geq h_{\text {top }}(\mathcal{U}) \text {. }
$$

We will now present a new result which will provide the fact that

$$
\sup _{\mu \in M_{T}(X)} \inf _{\alpha \succ U} h_{\mu}(\alpha)=h_{\text {top }}(\mathcal{U})
$$

thus completing the proof of a variational principle for $\mathcal{U}$.

We first need a universal version of the Rohlin lemma.

7.9. Proposition. Let $(X, T)$ be a (Polish) dynamical system and assume that there exists on $X$ a T-invariant aperiodic probability measure. Given a positive integer $n$ and a real number $\delta>0$ there exists a Borel subset $B \subset X$ such that the sets $B, T B, \ldots, T^{n-1} B$ are pairwise disjoint and for every aperiodic $T$-invariant probability measure $\mu \in M_{T}(X)$ we have $\mu\left(\bigcup_{j=0}^{n-1} T^{j} B\right)>1-\delta$.

Proof. Fix $N$ (it should be larger than $n / \delta$ for the required height $n$ and error $\delta$ ). The set of points that are periodic with period $\leq N$ is closed. Any point in the complement (which by our assumption is nonempty) has, by continuity, a neighborhood $U$ with $N$ disjoint forward iterates. There is a countable subcover $\left\{U_{m}\right\}$ of such sets since the space is Polish. Take $A_{1}=U_{1}$ as a base for a Kakutani sky-scraper

$$
\begin{gathered}
\left\{T^{j} A_{1}^{k}: j=0, \ldots, k-1 ; k=1,2, \ldots\right\}, \\
A_{1}^{k}=\left\{x \in A_{1}: r_{A_{1}}(x)=k\right\},
\end{gathered}
$$

where $r_{A_{1}}(x)$ is the first integer $j \geq 1$ with $T^{j} x \in A_{1}$. Next set

$$
B_{1}=\bigcup_{k \geq 1}^{[(k-n-1) / n]} \bigcup_{j=0}^{j n} A_{1}^{k}
$$

so that the sets $B_{1}, T B_{1}, \ldots, T^{n-1} B_{1}$ are pairwise disjoint.

Remove the full forward $T$ orbit of $U_{1}$ from the space and repeat to find $B_{2}$ using as a base for the next Kakutani sky-scraper $A_{2}$ defined as $U_{2}$ intersected with the part of $X$ not removed earlier. Proceed by induction to define the sequence $B_{i}, i=1,2, \ldots$ and set $B=\bigcup_{i=1}^{\infty} B_{i}$. By Poincaré recurrence for any aperiodic invariant measure we exhaust the whole space except for $n$ iterates of the union $A$ of the bases of the Kakutani sky-scrapers. By construction $A=\bigcup_{m=1}^{\infty} A_{m}$ has $N$ disjoint iterates so that $\mu(A) \leq 1 / N$ for every $\mu \in M_{T}(X)$. Thus $B, T B, \ldots, T^{n-1} B$ fill all but $n / N<\delta$ of the space uniformly over the aperiodic measures $\mu \in M_{T}(X)$.

Let $(X, T)$ be a dynamical system and $\mathcal{U}=\left\{U_{1}, U_{2}, \ldots U_{\ell}\right\}$ a finite open cover. We denote by $\mathcal{A}$ the collection of all finite Borel partitions $\alpha$ which refine $\mathcal{U}$, i.e. for every $A \in \alpha$ there is some $U \in \mathcal{U}$ with $A \subset U$. We set

$$
\check{h}(\mathcal{U})=\sup _{\mu \in M_{T}(X)} \inf _{\alpha \in \mathcal{A}} h_{\mu}(\alpha) \quad \text { and } \quad \hat{h}(\mathcal{U})=\inf _{\alpha \in \mathcal{A}} \sup _{\mu \in M_{T}(X)} h_{\mu}(\alpha) .
$$

7.10. Proposition. Let $(X, T)$ be a dynamical system, $\mathcal{U}=\left\{U_{1}, U_{2}, \ldots U_{\ell}\right\}$ a finite open cover, then 
1. $\check{h}(\mathcal{U}) \leq \hat{h}(\mathcal{U})$,

2. $\hat{h}(\mathcal{U}) \leq h_{\text {top }}(\mathcal{U})$.

Proof. 1. Given $\nu \in M_{T}(X)$ and $\alpha \in \mathcal{A}$ we obviously have $h_{\nu}(\alpha) \leq \sup _{\mu \in M_{T}(X)} h_{\mu}(\alpha)$. Thus

$$
\inf _{\alpha \in \mathcal{A}} h_{\nu}(\alpha) \leq \inf _{\alpha \in \mathcal{A}} \sup _{\mu \in M_{T}(X)} h_{\mu}(\alpha)=\hat{h}(\mathcal{U})
$$

and therefore also $\check{h}(\mathcal{U}) \leq \hat{h}(\mathcal{U})$.

2. Choose for $\epsilon>0$ an integer $N$ large enough so that there is a subcover $\mathcal{D} \subset$ $\mathcal{U}_{0}^{N-1}=\bigvee_{j=0}^{N-1} T^{-j} \mathcal{U}$ of cardinality $2^{N\left(h_{\mathrm{top}}(\mathcal{U})+\epsilon\right)}$. Apply Proposition [7.9 to find a set $B$ such that the sets $B, T B, \ldots, T^{N-1} B$ are pairwise disjoint and for every $T$-invariant Borel probability measure $\mu \in M_{T}(X)$ we have $\mu\left(\bigcup_{j=0}^{N-1} T^{j} B\right)>1-\delta$. Consider $\mathcal{D}_{B}=\{D \cap B: D \in \mathcal{D}\}$, the restriction of the cover $\mathcal{D}$ to $B$, and find a partition $\beta$ of $B$ which refines $\mathcal{D}_{B}$. Thus each element $P \in \beta$ has the form

$$
P=P_{i_{0}, i_{1}, \ldots, i_{N-1}} \subset\left(\bigcap_{j=0}^{N-1} T^{-j} U_{i_{j}}\right) \cap B,
$$

where $\bigcap_{j=0}^{N-1} T^{-j} U_{i_{j}}$ represents a typical element of $\mathcal{D}$. Next use the partition $\beta$ of $B$ to define a partition $\alpha=\left\{A_{i}: i=1, \ldots, \ell\right\}$ of $\bigcup_{j=0}^{N-1} T^{j} B$ by assigning to the set $A_{i}$ all sets of the form $T^{j} P_{i_{0}, i_{1}, \ldots, i_{j}, \ldots, i_{N-1}}$ where $i_{j}=i$ ( $j$ can be any number in $[0, N-1]$ ). On the remainder of the space $\alpha$ can be taken to be any partition refining $\mathcal{U}$.

Now if $N$ is large and $\delta$ small enough then

$$
h_{\mu}(\alpha) \leq h_{\text {top }}(\mathcal{U})+2 \epsilon .
$$

Here is a sketch of how one establishes this inequality. For $n>>N$ we will estimate $H_{\mu}\left(\alpha_{0}^{n-1}\right)$ by counting how many $(n, \alpha)$-names are needed to cover most of the space. We take $\delta>0$ so that $\sqrt{\delta}<<\epsilon$. Denote $E=B \cup T B \cup \cdots \cup T^{N-1} B$ (so that $\mu(E)>1-\delta)$. Define

$$
f(x)=\frac{1}{n} \sum_{i=0}^{n} \mathbf{1}_{E}\left(T^{i} x\right),
$$

and observe that $0 \leq f \leq 1$ and

$$
\int_{X} f(x) d \mu(x)>1-\delta
$$

since $T$ is measure preserving. Therefore $\int(1-f)<\delta$ and (Markov's inequality)

$$
\mu\{x:(1-f) \geq \sqrt{\delta}\} \leq \frac{1}{\sqrt{\delta}} \int(1-f) \leq \sqrt{\delta} .
$$

It follows that for points $x$ in $G=\{f>1-\sqrt{\delta}\}$, we have the property that $T^{i} x \in E$ for most $i$ in $[0, n]$.

Partition $G$ according to the values of $i$ for which $T^{i} x \in B$. This partition has at most

$$
\sum_{j \leq \frac{n}{N}}\left(\begin{array}{l}
n \\
j
\end{array}\right) \leq \frac{n}{N}\left(\begin{array}{c}
n \\
n / N
\end{array}\right)
$$

sets, a number which is exponentially small in $n$ (if $N$ is sufficiently large). 
For a fixed choice of these values the times when we are not in $E$ take only $n \sqrt{\delta}$ values and there we have $<l^{n \sqrt{\delta}}$ choices.

Finally when $T^{i} x \in B$ we have at most $2^{\left(N\left(h_{\mathrm{top}}(U)+\epsilon\right)\right)}$ names so that the total contribution is $<2^{\left(N\left(h_{\text {top }}(U)+\epsilon\right)\right) \frac{n}{N}}$.

Collecting these estimations we find that

$$
H\left(\alpha_{0}^{n-1}\right)<n\left(h_{\text {top }}(U)+2 \epsilon\right),
$$

whence (5). This completes the proof of the proposition.

We finally obtain:

7.11. Theorem (The variational principle for open covers). Let $(X, T)$ be a dynamical system, $\mathcal{U}=\left\{U_{1}, U_{2}, \ldots U_{k}\right\}$ a finite open cover and denote by $\mathcal{A}$ the collection of all finite Borel partitions $\alpha$ which refine $\mathcal{U}$, then

1. for every $\mu \in M_{T}(X) \inf _{\alpha \in \mathcal{A}} h_{\mu}(\alpha) \leq h_{\text {top }}(\mathcal{U})$, and

2. there exists an ergodic measure $\mu_{0} \in M_{T}(X)$ with $h_{\mu_{0}}(\alpha) \geq h_{\text {top }}(\mathcal{U})$ for every Borel partition $\alpha \in \mathcal{A}$.

3 .

$$
\check{h}(\mathcal{U})=\hat{h}(\mathcal{U})=h_{\mathrm{top}}(\mathcal{U})
$$

Proof. 1. This assertion can be formulated by the inequality $\breve{h}(\mathcal{U}) \leq h_{\text {top }}(\mathcal{U})$ and it follows by combining the two parts of Lemma 7.10 .

2. This is the content of Theorem 7.5 .

3. Combine assertions 1 and 2 .

7.5. Further results connecting topological and measure entropy. Given a topological dynamical system $(X, T)$ and a measure $\mu \in M_{T}(X)$, let $\pi:(X, \mathcal{X}, \mu, T) \rightarrow$ $(Z, Z, \eta, T)$ be the measure-theoretical Pinsker factor of $(X, X, \mu, T)$, and let $\mu=$ $\int_{Z} \mu_{z} d \eta(z)$ be the disintegration of $\mu$ over $(Z, \eta)$. Set

$$
\lambda=\int_{Z}\left(\mu_{z} \times \mu_{z}\right) d \eta(z)
$$

the relatively independent joining of $\mu$ with itself over $\eta$. Finally let $\Lambda_{\mu}=\operatorname{supp}(\lambda)$ be the topological support of $\lambda$ in $X \times X$. Although the Pinsker factor is, in general, only defined measure theoretically, the measure $\lambda$ is a well defined element of $M_{T \times T}(X \times$ $X)$. It was shown in Glasner [31] that $E_{\mu}=\Lambda_{\mu} \backslash \Delta$.

7.12. Theorem. Let $(X, T)$ be a topological dynamical system and let $\mu \in M_{T}(X)$.

1. $E_{\mu}=\Lambda_{\mu} \backslash \Delta$ and $\Lambda_{\mu}=E_{\mu} \cup\{(x, x): x \in \operatorname{supp}(\mu)\}$.

2. $\operatorname{cls} E_{\mu} \subset \Lambda_{\mu}$.

3. If $\mu$ is ergodic with positive entropy then $\operatorname{cls} E_{\mu}=\Lambda_{\mu}$.

One consequence of this characterization of the set of $\mu$-entropy pairs is a description of the set of entropy pairs of a product system. Recall that an $E$-system is a system for which there exists a probability invariant measure with full support.

7.13. Corollary. Let $\left(X_{1}, T\right)$ and $\left(X_{2}, T\right)$ be two topological E-systems then:

1. $E_{X_{1} \times X_{2}}=\left(E_{X_{1}} \times E_{X_{2}}\right) \cup\left(E_{X_{1}} \times \Delta_{X_{2}}\right) \cup\left(\Delta_{X_{1}} \times E_{X_{2}}\right)$. 
2. The product of two UPE systems is UPE.

Another consequence is:

7.14. Corollary. Let $(X, T)$ be a topological dynamical system, $P$ the proximal relation on $X$. Then:

1. For every $T$-invariant ergodic measure $\mu$ of positive entropy the set $P \cap E_{\mu}$ is residual in the $G_{\delta}$ set $E_{\mu}$ of $\mu$ entropy pairs.

2. When $E_{X} \neq \emptyset$ the set $P \cap E_{X}$ is residual in the $G_{\delta}$ set $E_{X}$ of topological entropy pairs.

Given a dynamical system $(X, T)$, a pair $\left(x, x^{\prime}\right) \in X \times X$ is called a Li-Yorke pair if it is a proximal pair but not an asymptotic pair. A set $S \subseteq X$ is called scrambled if any pair of distinct points $\{x, y\} \subseteq S$ is a Li-Yorke pair. A dynamical system $(X, T)$ is called chaotic in the sense of $\mathbf{L i}$ and Yorke if there is an uncountable scrambled set. In [1] Theorem $\mathbf{7 . 1 2}$ is applied to solve the question whether positive topological entropy implies Li-Yorke chaos as follows.

7.15. Theorem. Let $(X, T)$ be a topological dynamical system.

1. If $(X, T)$ admits a T-invariant ergodic measure $\mu$ with respect to which the measure preserving system $(X, X, \mu, T)$ is not measure distal then $(X, T)$ is Li-Yorke chaotic.

2. If $(X, T)$ has positive topological entropy then it is Li-Yorke chaotic.

In 14] Blanchard, Host and Ruette show that in positive entropy systems there are also many asymptotic pairs.

7.16. Theorem. Let $(X, T)$ be a topological dynamical system with positive topological entropy. Then

1. The set of points $x \in X$ for which there is some $x^{\prime} \neq x$ with $\left(x, x^{\prime}\right)$ an asymptotic pair, has measure 1 for every invariant probability measure on $X$ with positive entropy.

2. There exists a probability measure $\nu$ on $X \times X$ such that $\nu$ a.e. pair $\left(x, x^{\prime}\right)$ is Li-Yorke and positively asymptotic; or more precisely for some $\delta>0$

$$
\begin{gathered}
\lim _{n \rightarrow+\infty} d\left(T^{n} x, T^{n} x^{\prime}\right)=0, \quad \text { and } \\
\liminf _{n \rightarrow+\infty} d\left(T^{-n} x, T^{-n} x^{\prime}\right)=0, \quad \limsup _{n \rightarrow+\infty} d\left(T^{-n} x, T^{-n} x^{\prime}\right) \geq \delta .
\end{gathered}
$$

7.6. Topological determinism and zero entropy. Following [54] call a dynamical system $(X, T)$ deterministic if every $T$-factor is also a $T^{-1}$-factor. In other words every closed equivalence relation $R \subset X \times X$ which has the property $T R \subset R$ also satisfies $T^{-1} R \subset R$. It is not hard to see that an equivalent condition is as follows. For every continuous real valued function $f \in C(X)$ the function $f \circ T^{-1}$ is contained in the smallest closed subalgebra $\mathcal{A} \subset C(X)$ which contains the constant function 1 and the collection $\left\{f \circ T^{n}: n \geq 0\right\}$. The folklore question whether the latter condition implies zero entropy was open for awhile. Here we note that the affirmative answer is a direct consequence of Theorem 7.16 (see also [54]). 
7.17. Proposition. Let $(X, T)$ be a topological dynamical system such that there exists $a \delta>0$ and a pair $\left(x, x^{\prime}\right) \in X \times X$ as in Theorem 17.16.2. Then $(X, T)$ is not deterministic.

Proof. Set

$$
R=\left\{\left(T^{n} x, T^{n} x^{\prime}\right): n \geq 0\right\} \cup\left\{\left(T^{n} x^{\prime}, T^{n} x\right): n \geq 0\right\} \cup \Delta .
$$

Clearly $R$ is a closed equivalence relation which is $T$-invariant but not $T^{-1}$-invariant.

7.18. Corollary. A topologically deterministic dynamical system has zero entropy.

Proof. Let $(X, T)$ be a topological dynamical system with positive topological entropy; by Theorem [7.16.2. and Proposition [7.17] it is not deterministic. 


\section{Part 2. Meeting grounds}

\section{UNIQUE ERGODICITY}

The topological system $(X, T)$ is called uniquely ergodic if $M_{T}(X)$ consists of a single element $\mu$. If in addition $\mu$ is a full measure (i.e. supp $\mu=X$ ) then the system is called strictly ergodic (see 46, Section 4.3]). Since the ergodic measures are characterized as the extreme points of the Choquet simplex $M_{T}(X)$, it follows immediately that a uniquely ergodic measure is ergodic. For a while it was believed that strict ergodicity — which is known to imply some strong topological consequences (like in the case of $\mathbb{Z}$-systems, the fact that every point of $X$ is a generic point and moreover that the convergence of the ergodic sums $\mathbb{A}_{n}(f)$ to the integral $\int f d \mu, f \in C(X)$ is uniform ) - entails some severe restrictions on the measure-theoretical behavior of the system. For example, it was believed that unique ergodicity implies zero entropy. Then, at first some examples were produced to show that this need not be the case. Furstenberg in 23] and Hahn and Katznelson in [44] gave examples of uniquely ergodic systems with positive entropy. Later in 1970 R. I. Jewett surprised everyone with his outstanding result: every weakly mixing measure preserving $\mathbb{Z}$-system has a strictly ergodic model, [50. This was strengthened by Krieger [59] who showed that even the weak mixing assumption is redundant and that the result holds for every ergodic $\mathbb{Z}$-system.

We recall the following well known characterizations of unique ergodicity (see [35, Theorem 4.9]).

8.1. Proposition. Let $(X, T)$ be a topological system. The following conditions are equivalent.

1. $(X, T)$ is uniquely ergodic.

2. $C(X)=\mathbb{R}+\bar{B}$, where $B=\{g-g \circ T: g \in C(X)\}$.

3. For every continuous function $f \in C(X)$ the sequence of functions

$$
\mathbb{A}_{n} f(x)=\frac{1}{n} \sum_{j=0}^{n-1} f\left(T^{j} x\right)
$$

converges uniformly to a constant function.

4. For every continuous function $f \in C(X)$ the sequence of functions $\mathbb{A}_{n}(f)$ converges pointwise to a constant function.

5. For every function $f \in A$, for a collection $A \subset C(X)$ which linearly spans a uniformly dense subspace of $C(X)$, the sequence of functions $\mathbb{A}_{n}(f)$ converges pointwise to a constant function.

Given an ergodic dynamical system $\mathbf{X}=(X, X, \mu, T)$ we say that the system $\hat{\mathbf{X}}=$ $(\hat{X}, \hat{X}, \hat{\mu}, T)$ is a topological model (or just a model) for $\mathbf{X}$ if $(\hat{X}, T)$ is a topological system, $\hat{\mu} \in M_{T}(\hat{X})$ and the systems $\mathbf{X}$ and $\hat{\mathbf{X}}$ are measure theoretically isomorphic. Similarly we say that $\hat{\pi}: \hat{\mathbf{X}} \rightarrow \hat{\mathbf{Y}}$ is a topological model for $\pi: \mathbf{X} \rightarrow \mathbf{Y}$ when $\hat{\pi}$ is a topological factor map and there exist measure theoretical isomorphisms $\phi$ and $\psi$ 
such that the diagram

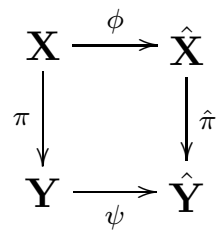

is commutative.

\section{The Relative JeWetT-Krieger theorem}

In this subsection we will prove the following generalization of the Jewett-Krieger theorem (see [46, Theorem 4.3.10]).

9.1. Theorem. If $\pi: \mathbf{X}=(X, X, \mu, T) \rightarrow \mathbf{Y}=(Y, y, \nu, T)$ is a factor map with $\mathbf{X}$ ergodic and $\hat{\mathbf{Y}}$ is a uniquely ergodic model for $\mathbf{Y}$ then there is a uniquely ergodic model $\hat{\mathbf{X}}$ for $\mathbf{X}$ and a factor map $\hat{\pi}: \hat{\mathbf{X}} \rightarrow \hat{\mathbf{Y}}$ which is a model for $\pi: \mathbf{X} \rightarrow \mathbf{Y}$.

In particular, taking $\mathbf{Y}$ to be the trivial one point system we get:

9.2. Theorem. Every ergodic system has a uniquely ergodic model.

Several proofs have been given of this theorem, e.g. see [17] and [7. We will sketch a proof which will serve the relative case as well.

Proof of theorem 9.1. A key notion for this proof is that of a uniform partition whose importance in this context was emphasized by G. Hansel and J.-P. Raoult, 45].

9.3. Definition. A set $B \in \mathcal{X}$ is uniform if

$$
\lim _{N \rightarrow \infty} \operatorname{ess}^{-\sup _{x}}\left|\frac{1}{N} \sum_{0}^{N-1} 1_{B}\left(T^{i} x\right)-\mu(B)\right|=0 .
$$

A partition $\mathcal{P}$ is uniform if, for all $N$, every set in $\bigvee_{-N}^{N} T^{-i \mathcal{P}}$ is uniform.

The connection between uniform sets, partitions and unique ergodicity lies in Proposition 8.1. It follows easily from that proposition that if $\mathcal{P}$ is a uniform partition, say into the sets $\left\{P_{1}, P_{2}, \ldots, P_{a}\right\}$, and we denote by $\mathcal{P}$ also the mapping that assigns to $x \in X$, the index $1 \leq i \leq a$ such that $x \in P_{i}$, then we can map $X$ to $\{1,2, \ldots, a\}^{\mathbb{Z}}=A^{\mathbb{Z}}$ by:

$$
\pi(x)=\left(\ldots, \mathcal{P}\left(T^{-1} x\right), \mathcal{P}(x), \mathcal{P}(T x), \ldots, \mathcal{P}\left(T^{n} x\right), \ldots\right) .
$$

Pushing forward the measure $\mu$ by $\pi$, gives $\pi \circ \mu$ and the closed support of this measure will be a closed shift invariant subset, say $E \subset A^{\mathbb{Z}}$. Now the indicator functions of finite cylinder sets span the continuous functions on $E$, and the fact that $\mathcal{P}$ is a uniform partition and Proposition 8.1 combine to establish that ( $E$, shift) is uniquely ergodic. This will not be a model for $(X, X, \mu, T)$ unless $\bigvee_{-\infty}^{\infty} T^{-i} \mathcal{P}=X$ modulo null sets, but in any case this does give a model for a nontrivial factor of $X$.

Our strategy for proving Theorem 9.2 is to first construct a single nontrivial uniform partition. Then this partition will be refined more and more via uniform partitions until we generate the entire $\sigma$-algebra $\mathcal{X}$. Along the way we will be showing how one can prove a relative version of the basic Jewett-Krieger theorem. Our main tool is the use 
of Rohlin towers. These are sets $B \in \mathcal{X}$ such that for some $N, B, T B, \ldots, T^{N-1} B$ are disjoint while $\bigcup_{0}^{N-1} T^{i} B$ fill up most of the space. Actually we need Kakutani-Rohlin towers, which are like Rohlin towers but fill up the whole space. If the transformation does not have rational numbers in its point spectrum this is not possible with a single height, but two heights that are relatively prime, like $N$ and $N+1$ are certainly possible. Here is one way of doing this. The ergodicity of $(X, X, \mu, T)$ with $\mu$ non atomic easily yields, for any $n$, the existence of a positive measure set $B$, such that

$$
T^{i} B \cap B=\emptyset \quad, \quad i=1,2, \ldots, n .
$$

With $N$ given, choose $n \geq 10 \cdot N^{2}$ and find $B$ that satisfies the above. It follows that the return time

$$
r_{B}(x)=\inf \left\{i>0: T^{i} x \in B\right\}
$$

is greater than $10 \cdot N^{2}$ on $B$. Let

$$
B_{\ell}=\left\{x: r_{B}(x)=\ell\right\} .
$$

Since $\ell$ is large (if $B_{\ell}$ is nonempty) one can write $\ell$ as a positive combination of $N$ and $N \times 1$, say

$$
\ell=N u_{\ell}+(N+1) v_{\ell} .
$$

Now divide the column of sets $\left\{T^{i} B_{\ell}: 0 \leq i<\ell\right\}$ into $u_{\ell^{-}}$blocks of size $N$ and $v_{\ell^{-}}$ blocks of size $N+1$ and mark the first layer of each of these blocks as belonging to $C$. Taking the union of these marked levels $\left(T^{i} B_{\ell}\right.$ for suitably chosen $i$ ) over the various columns gives us a set $C$ such that $r_{C}$ takes only two values - either $N$ or $N+1$ as required.

It will be important for us to have at our disposal K-R towers like this such that the columns of say the second K-R tower are composed of entire subcolumns of the earlier one. More precisely we want the base $C_{2}$ to be a subset of $C_{1}$ - the base of the first tower. Although we are not sure that this can be done with just two column heights we can guarantee a bound on the number of columns that depends only on the maximum height of the first tower. Let us define formally:

9.4. Definition. A set $C$ will be called the base of a bounded K-R tower if for some $N, \bigcup_{0}^{N-1} T^{i} C=X$ up to a $\mu$-null set. The least $N$ that satisfies this will be called the height of $C$, and partitioning $C$ into sets of constancy of $r_{C}$ and viewing the whole space $X$ as a tower over $C$ will be called the K-R tower with columns the sets $\left\{T^{i} C_{\ell}: 0 \leq i<\ell\right\}$ for $C_{\ell}=\left\{x \in C: r_{C}(x)=\ell\right\}$.

Our basic lemma for nesting these K-R towers is:

9.5. Lemma. Given a bounded $K-R$ tower with base $C$ and height $N$, for any $n$ sufficiently large there is a bounded $K-R$ tower with base $D$ contained in $C$ whose column heights are all at least $n$ and at most $n+4 N$.

Proof. We take an auxiliary set $B$ such that $T^{i} B \cap B=\emptyset$ for all $0<i<10(n+2 N)^{2}$ and look at the unbounded (in general) K-R tower over $B$. Using the ergodicity it is easy to arrange that $B \subset C$. Now let us look at a single column over $B_{m}$, with $m \geq 10(n+2 N)^{2}$. We try to put down blocks of size $n+2 N$ and $n+2 N+1$, to fill up the tower. This can certainly be done but we want our levels to belong to $C$. We can refine the column over $B_{m}$ into a finite number of columns so that each level is either 
entirely within $C$ or in $X \backslash C$. This is done by partitioning the base $C$ according to the finite partition:

$$
\bigcap_{i=0}^{m-1} T^{-1}\{C, X \backslash C\} .
$$

Then we move the edge of each block to the nearest level that belongs to $C$. The fact that the height of $C$ is $N$ means that we do not have to move any level more than $N-1$ steps, and so at most we lose $2 N-2$ or gain that much thus our blocks, with bases now all in $C$, have size in the interval $[n, n+4 N]$ as required.

It is clear that this procedure can be iterated to give an infinite sequence of nested $\mathrm{K}-\mathrm{R}$ towers with a good control on the variation in the heights of the columns. These can be used to construct uniform partitions in a pretty straightforward way, but we need one more lemma which strengthens slightly the ergodic theorem. We will want to know that when we look at a bounded K-R tower with base $C$ and with minimum column height sufficiently large that for most of the fibers of the towers (that is for $\left.x \in C,\left\{T^{i} x: 0 \leq i<r_{C}(x)\right\}\right)$ the ergodic averages of some finite set of functions are close to the integrals of the functions. It would seem that there is a problem because the base of the tower is a set of very small measure (less than $1 /$ min column height) and it may be that the ergodic theorem is not valid there. However, a simple averaging argument using an intermediate size gets around this problem. Here is the result which we formulate for simplicity for a single function $f$ :

9.6. Lemma. Let $f$ be a bounded function and $(X, X, \mu, T)$ ergodic. Given $\epsilon>0$, there is an $n_{0}$, such that if a bounded $K-R$ tower with base $C$ has minimum column height at least $n_{0}$, then those fibers over $x \in C:\left\{T^{i} x: 0 \leq i<r_{C}(x)\right\}$ that satisfy

$$
\left|\frac{1}{r_{C}(x)} \sum_{i=0}^{r_{C}(x)-1} f\left(T^{i} x\right)-\int_{X} f d \mu\right|<\epsilon
$$

fill up at least $1-\epsilon$ of the space.

Proof. Assume without loss of generality that $|f| \leq 1$. For a $\delta$ to be specified later find an $N$ such that the set of $y \in X$ which satisfy

$$
\left|\frac{1}{N} \sum_{0}^{N-1} f\left(T^{i} y\right)-\int f d \mu\right|<\delta
$$

has measure at least $1-\delta$. Let us denote the set of $y$ that satisfy (6) by $E$. Suppose now that $n_{0}$ is large enough so that $N / n_{0}$ is negligible - say at most $\delta$. Consider a bounded K-R tower with base $C$ and with minimum column height greater than $n_{0}$. For each fiber of this tower, let us ask what is the fraction of its points that lie in $E$. Those fibers with at least a $\sqrt{\delta}$ fraction of its points not in $E$ cannot fill up more than a $\sqrt{\delta}$ fraction of the space, because $\mu(E)>1-\delta$.

Fibers with more than $1-\sqrt{\delta}$ of its points lying in $E$ can be divided into disjoint blocks of size $N$ that cover all the points that lie in $E$. This is done by starting at $x \in C$, and moving up the fiber, marking the first point in $E$, skipping $N$ steps and continuing to the next point in $E$ until we exhaust the fiber. On each of these $N$ blocks the average of $f$ is within $\delta$ of its integral, and since $|f| \leq 1$ if $\sqrt{\delta}<\epsilon / 10$ this will guarantee that the average of $f$ over the whole fiber is within $\epsilon$ of its integra. 
We are now prepared to construct uniform partitions. Start with some fixed nontrivial partition $\mathcal{P}_{0}$. By Lemma 9.6, for any tall enough bounded $\mathrm{K}-\mathrm{R}$ tower at least $9 / 10$ of the columns will have the 1 -block distribution of each $\mathcal{P}_{0}$-name within $\frac{1}{10}$ of the actual distribution. We build a bounded K-R tower with base $C_{1}(1)$ and heights $N_{1}, N_{1}+1$ with $N_{1}$ large enough for this to be valid. It is clear that we can modify $\mathcal{P}_{0}$ to $\mathcal{P}_{1}$ on the bad fibers so that now all fibers have a distribution of 1-blocks within $\frac{1}{10}$ of a fixed distribution. We call this new partition $\mathcal{P}_{1}$. Our further changes in $\mathcal{P}_{1}$ will not change the $N_{1}, N_{1}+1$ blocks that we see on fibers of a tower over our ultimate $C_{1}$. Therefore, we will get a uniformity on all blocks of size $100 N_{1}$. The 100 is to get rid of the edge effects since we only know the distribution across fibers over points in $C_{1}(1)$.

Next we apply Lemma 9.6 to the 2-blocks in $\mathcal{P}_{1}$ with $1 / 100$. We choose $N_{2}$ so large that $N_{1} / N_{2}$ is negligible and so that any uniform K-R tower with height at least $N_{2}$ has for at least $99 / 100$ of its fibers a distribution of 2-blocks within 1/100 of the global $\mathcal{P}_{1}$ distribution. Apply Lemma 9.5 to find a uniform K-R tower with base $C_{2}(2) \subset C_{1}(1)$ such that its column heights are between $N_{2}$ and $N_{2}+4 N_{1}$. For the fibers with good $\mathcal{P}_{1}$ distribution we make no change. For the others, we copy on most of the fiber (except for the top $10 \cdot N_{1}^{2}$ levels) the corresponding $\mathcal{P}_{1}$-name from one of the good columns. In this copying we also copy the $C_{1}(1)$-name so that we preserve the blocks. The final $10 \cdot N_{1}^{2}$ spaces are filled in with $N_{1}, N_{1}+1$ blocks. This gives us a new base for the first tower that we call $C_{1}(2)$, and a new partition $\mathcal{P}_{2}$. The features of $\mathcal{P}_{2}$ are that all its fibers over $C_{1}(2)$ have good (up to 1/10) 1-block distribution, and all its fibers over $C_{2}(2)$ have good (up to 1/100) 2-block distributions. These will not change in the subsequent steps of the construction.

Note too that the change from $C_{1}(1)$, to $C_{1}(2)$, could have been made arbitrarily small by choosing $N_{2}$ sufficiently large.

There is one problem in trying to carry out the next step and that is, the filling in of the top relatively small portion of the bad fibers after copying most of a good fiber. We cannot copy an exact good fiber because it is conceivable that no fiber with the precise height of the bad fiber is good. The filling in is possible if the column heights of the previous level are relatively prime. This was the case in step 2, because in step 1 we began with a K-R tower heights $N_{1}, N_{1}+1$. However, Lemma 9.5 does not guarantee relatively prime heights. This is automatically the case if there is no rational spectrum. If there are only a finite number of rational points in the spectrum then we could have made our original columns with heights $L N_{1}, L\left(N_{1}+1\right)$ with $L$ being the highest power so that $T^{L}$ is not ergodic and then worked with multiples of $L$ all the time. If the rational spectrum is infinite then we get an infinite group rotation factor and this gives us the required uniform partition without any further work.

With this point understood it is now clear how one continues to build a sequence of partitions $\mathcal{P}_{n}$ that converge to $\mathcal{P}$ and $C_{i}(k) \rightarrow C_{i}$ such that the $\mathcal{P}$-names of all fibers over points in $C_{i}$ have a good (up to $1 / 10^{i}$ ) distribution of $i$-blocks. This gives the uniformity of the partition $\mathcal{P}$ as required and establishes

9.7. Proposition. Given any $\mathcal{P}_{0}$ and any $\epsilon>0$ there is a uniform partition $\mathcal{P}$ such that $d\left(\mathcal{P}_{0}, \mathcal{P}\right)<\epsilon$ in the $\ell_{1}$-metric on partitions. 
As we have already remarked the uniform partition that we have constructed gives us a uniquely ergodic model for the factor system generated by this partition. We need now a relativized version of the construction we have just carried out. We formulate this as follows:

9.8. Proposition. Given a uniform partition $\mathcal{P}$ and an arbitrary partition $\mathcal{Q}_{0}$ that refines $\mathcal{P}$, for any $\epsilon>0$ there is a uniform partition $\mathcal{Q}$ that also refines $\mathcal{P}$ and satisfies

$$
\left\|Q_{0}-\mathcal{Q}\right\|_{1}<\epsilon
$$

Even though we write things for finite alphabets, everything makes good sense for countable partitions as well and the arguments need no adjusting. However, the metric used to compare partitions becomes important since not all metrics on $\ell_{1}$ are equivalent. We use always:

$$
\|Q-\bar{Q}\|_{1}=\sum_{j} \int_{X}\left|1_{Q_{j}}-1_{\bar{Q}_{j}}\right| d \mu
$$

where the partitions $Q$ and $\bar{Q}$ are ordered partitions into sets $\left\{Q_{j}\right\},\left\{\bar{Q}_{j}\right\}$ respectively. We also assume that the $\sigma$-algebra generated by the partition $\mathcal{P}$ is nonatomic otherwise there is no real difference between what we did before and what has to be done here.

We will try to follow the same proof as before. The problem is that when we redefine $Q_{0}$ to $Q$ we are not allowed to change the $\mathcal{P}$-part of the name of points. That greatly restricts us in the kind of names we are allowed to copy on columns of K-R towers and it is not clear how to proceed. The way to overcome the difficulty is to build the K-R towers inside the uniform algebra generated by $\mathcal{P}$. This being done we look, for example, at our first tower and the first change we wish to make in $Q_{0}$. We divide the fibers into a finite number of columns according to the height and according to the $\mathcal{P}$-name.

Next each of these is divided into subcolumns, called $\mathcal{Q}_{0}$-columns, according to the $\mathcal{Q}_{0}$-names of points. If a $\mathcal{P}$-column has some good (i.e. good 1-block distribution of $Q_{0}$-names) $\mathcal{Q}_{0}$-subcolumn it can be copied onto all the ones that are not good. Next notice that a $\mathcal{P}$-column that contains not even one good $\mathcal{Q}_{0}$-name is a set defined in the uniform algebra. Therefore if these sets have small measure then for some large enough $N$, uniformly over the whole space, we will not encounter these bad columns too many times.

In brief the solution is to change the nature of the uniformity. We do not make all of the columns of the K-R tower good - but we make sure that the bad ones are seen infrequently, uniformly over the whole space. With this remark the proof of the proposition is easily accomplished using the same nested K-R towers as before - but inside the uniform algebra.

Finally the J-K theorem is established by constructing a refining sequence of uniform partitions and looking at the inverse limit of the corresponding topological spaces. Notice that if $\mathcal{Q}$ refines $\mathcal{P}$, and both are uniform, then there is a natural homeomorphism from $X_{\mathcal{Q}}$ onto $X_{\mathcal{P}}$. The way in which the theorem is established also yields a proof of the relative $\mathrm{J}-\mathrm{K}$ theorem, Theorem 9.1 
Using similar methods E. Lehrer 61] shows that in the Jewett-Krieger theorem one can find, for any ergodic system, a strictly ergodic model which is topologically mixing.

\section{Models For Other COMmUtative Diagrams}

One can describe Theorem 9.1 as asserting that every diagram of ergodic systems of the form $\mathbf{X} \rightarrow \mathbf{Y}$ has a strictly ergodic model. What can we say about more complicated commutative diagrams? A moments reflection will show that a repeated application of Theorem 9.1 proves the first assertion of the following theorem.

10.1. Theorem. Any commutative diagram in the category of ergodic $\mathbb{Z}$ dynamical systems with the structure of an inverted tree, i.e. no portion of it looks like

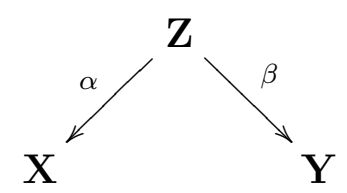

has a strictly ergodic model. On the other hand there exists a diagram of the form (17) that does not admit a strictly ergodic model.

For the proof of the second assertion we need the following theorem.

10.2. Theorem. If $(Z, \eta, T)$ is a strictly ergodic system and $(Z, T) \stackrel{\alpha}{\rightarrow}(X, T)$ and $(Z, T) \stackrel{\beta}{\rightarrow}(Y, T)$ are topological factors such that $\alpha^{-1}(U) \cap \beta^{-1}(V) \neq \emptyset$ whenever $U \subset X$ and $V \subset Y$ are nonempty open sets, then the measure-preserving systems $\mathbf{X}=(X, X, \mu, T)$ and $\mathbf{Y}=(Y, y, \nu, T)$ are measure-theoretically disjoint. In particular this is the case if the systems $(X, T)$ and $(Y, T)$ are topologically disjoint.

Proof. It suffices to show that the map $\alpha \times \beta: Z \rightarrow X \times Y$ is onto since this will imply that the topological system $(X \times Y, T)$ is strictly ergodic. We establish this by showing that the measure $\lambda=(\alpha \times \beta)_{*}(\eta)$ (a joining of $\mu$ and $\nu$ ) is full; i.e. that it assigns positive measure to every set of the form $U \times V$ with $U$ and $V$ as in the statement of the theorem. In fact, since by assumption $\eta$ is full we have

$$
\lambda(U \times V)=\eta\left((\alpha \times \beta)^{-1}(U \times V)\right)=\eta\left(\alpha^{-1}(U) \cap \beta^{-1}(V)\right)>0 .
$$

This completes the proof of the first assertion. The second follows since topological disjointness of $(X, T)$ and $(Y, T)$ implies that $\alpha \times \beta: Z \rightarrow X \times Y$ is onto.

Proof of Theorem Theorem 10.1. We only need to prove the last assertion. Take $\mathbf{X}=$ $\mathbf{Y}$ to be any nontrivial weakly mixing system, then $\mathbf{X} \times \mathbf{X}$ is ergodic and the diagram

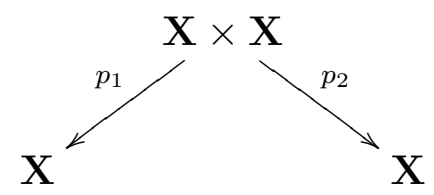

is our counter example. In fact if (17) is a uniquely ergodic model in this situation then it is easy to establish that the condition in Theorem 10.2 is satisfied and we 
apply this theorem to conclude that $\mathbf{X}$ is disjoint from itself. Since in a nontrivial system $\mu \times \mu$ and gr $(\mu$, id) are different ergodic joinings, this contradiction proves our assertion.

\section{The Furstenberg-Weiss almost 1-1 extension theorem}

It is well known that in a topological measure space one can have sets that are large topologically but small in the sense of the measure. In topological dynamics when $(X, T)$ is a factor of $(Y, T)$ and the projection $\pi: Y \rightarrow X$ is one to one on a topologically large set (i.e. the complement of a set of first category), one calls $(Y, T)$ an almost 1-1 extension of $(X, T)$ and considers the two systems to be very closely related. Nonetheless, in view of the opening sentence, it is possible that the measure theory of $(Y, T)$ will be quite different from the measure theory of $(X, T)$. The following theorem realizes this possibility in an extreme way (see [28]).

11.1. Theorem. Let $(X, T)$ be a non-periodic minimal dynamical system, and let $\pi$ : $Y \rightarrow X$ be an extension of $(X, T)$ with $(Y, T)$ topologically transitive and $Y$ a compact metric space. Then there exists an almost 1-1 minimal extension, $\bar{\pi}:(\bar{Y}, T) \rightarrow(X, T)$ and a Borel subset $Y_{0} \subset Y$ with a Borel measurable map $\theta: Y_{0} \rightarrow \bar{Y}$ satisfying (1) $\theta T=T \theta$, (2) $\pi \theta=\pi$, (3) $\theta$ is 1-1 on $Y_{0}$, (4) $\mu\left(Y_{0}\right)=1$ for any $T$-invariant measure $\mu$ on $Y$.

In words, one can find an almost 1-1 minimal extension of $X$ such that the measure theoretic structure is as rich as that of an arbitrary topologically transitive extension of $X$.

An almost 1-1 extension of a minimal equicontinuous system is called an almost automorphic system. The next corollary demonstrates the usefulness of this modelling theorem. Other applications appeared e.g. in [40] and [19].

11.2. Corollary. Let $(X, X, \mu, T)$ be an ergodic measure preserving transformation with infinite point spectrum defined by $(G, \rho)$ where $G$ is a compact monothetic group

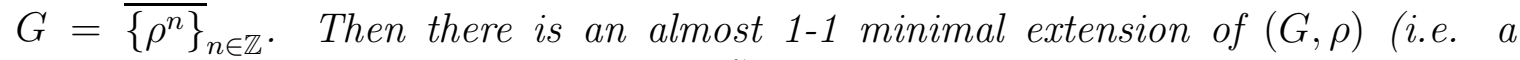
minimal almost automorphic system), $(\tilde{Z}, \sigma)$ and an invariant measure $\nu$ on $Z$ such that $(Z, \sigma, \nu)$ is isomorphic to $(X, X, \mu, T)$.

\section{CAntor minimal REPRESEntations}

A Cantor minimal dynamical system is a minimal topological system $(X, T)$ where $X$ is the Cantor set. Two Cantor minimal systems $(X, T)$ and $(Y, S)$ are called orbit equivalent $(\mathrm{OE})$ if there exists a homeomorphism $F: X \rightarrow Y$ such that $F\left(\mathcal{O}_{T}(x)\right)=$ $\mathcal{O}_{S}(F x)$ for every $x \in X$. Equivalently: there are functions $n: X \rightarrow \mathbb{Z}$ and $m: X \rightarrow \mathbb{Z}$ such that for every $x \in X F(T x)=S^{n(x)}(F x)$ and $F\left(T^{m(x)}\right)=S(F x)$. An old result of M. Boyle implies that the requirement that, say, the function $n(x)$ be continuous already implies that the two systems are flip conjugate; i.e. $(Y, S)$ is isomorphic either to $(X, T)$ or to $\left(X, T^{-1}\right)$. However, if we require that both $n(x)$ and $m(x)$ have at most one point of discontinuity we get the new and, as it turns out, useful notion of strong orbit equivalence (SOE). A complete characterization of both $\mathrm{OE}$ and SOE of 
Cantor minimal systems was obtained by Giordano Putnam and Skau [30] in terms of an algebraic invariant of Cantor minimal systems called the dimension group. (See 34] for a review of these results.)

We conclude this section with the following remarkable theorems, due to N. Ormes 67, which simultaneously generalize the theorems of Jewett and Krieger and a theorem of Downarowicz 18] which, given any Choquet simplex $Q$, provides a Cantor minimal system $(X, T)$ with $M_{T}(X)$ affinely homeomorphic with $Q$. (See also Downarowitcz and Serafin [20], and Boyle and Downarowicz [15].)

12.1. Theorem. $\quad$ 1. Let $(\Omega, \mathcal{B}, \nu, S)$ be an ergodic, non-atomic, probability measure preserving, dynamical system. Let $(X, T)$ be a Cantor minimal system such that whenever $\exp (2 \pi i / p)$ is a (topological) eigenvalue of $(X, T)$ for some $p \in \mathbb{N}$ it is also a (measurable) eigenvalue of $(\Omega, \mathcal{B}, \nu, S)$. Let $\mu$ be any element of the set of extreme points of $M_{T}(X)$. Then, there exists a homeomorphism $T^{\prime}: X \rightarrow X$ such that (i) $T$ and $T^{\prime}$ are strong orbit equivalent, (ii) $(\Omega, \mathcal{B}, \nu, S)$ and $\left(X, X, \mu, T^{\prime}\right)$ are isomorphic as measure preserving dynamical systems.

2. Let $(\Omega, \mathcal{B}, \nu, S)$ be an ergodic, non-atomic, probability measure preserving, $d y$ namical system. Let $(X, T)$ be a Cantor minimal system and $\mu$ any element of the set of extreme points of $M_{T}(X)$. Then, there exists a homeomorphism $T^{\prime}: X \rightarrow X$ such that (i) $T$ and $T^{\prime}$ are orbit equivalent, (ii) $(\Omega, \mathcal{B}, \nu, S)$ and $\left(X, X, \mu, T^{\prime}\right)$ are isomorphic as measure preserving dynamical systems.

3. Let $(\Omega, \mathcal{B}, \nu, S)$ be an ergodic, non-atomic, probability measure preserving $d y$ namical system. Let $Q$ be any Choquet simplex and $q$ an extreme point of $Q$. Then there exists a Cantor minimal system $(X, T)$ and an affine homeomorphism $\phi: Q \rightarrow M_{T}(X)$ such that, with $\mu=\phi(q),(\Omega, \mathcal{B}, \nu, S)$ and $(X, X, \mu, T)$ are isomorphic as measure preserving dynamical systems.

\section{OTHER RELATED THEOREMS}

Let us mention a few more striking representation results.

For the first one recall that a topological dynamical system $(X, T)$ is said to be prime if it has no non-trivial factors. A similar definition can be given for measure preserving systems. There it is easy to see that a prime system $(X, X, \mu, T)$ must have zero entropy. It follows from a construction in [75] that the same holds for topological entropy, namely any system $(X, T)$ with positive topological entropy has non-trivial factors. In 84 it is shown that any ergodic zero entropy dynamical system has a minimal model $(X, T)$ with the property that any pair of points $(u, v)$ not on the same orbit has a dense orbit in $X \times X$. Such minimal systems are necessarily prime, and thus we have the following result:

13.1. Theorem. An ergodic dynamical system has a topological, minimal, prime model iff it has zero entropy.

The second theorem, due to Glasner and Weiss [40], treats the positive entropy systems.

13.2. Theorem. An ergodic dynamical system has a strictly ergodic, UPE model iff it has positive entropy. 
We also have the following surprising result which is due to Weiss [83].

13.3. Theorem. There exists a minimal metric dynamical system $(X, T)$ with the property that for every ergodic probability measure preserving system $(\Omega, \mathcal{B}, \mu, S)$ there exists a $T$-invariant Borel probability measure $\nu$ on $X$ such that the systems $(\Omega, \mathcal{B}, \mu, S)$ and $(X, X, \nu, T)$ are isomorphic.

In 64 E. Lindenstrauss proves the following:

13.4. Theorem. Every ergodic measure distal $\mathbb{Z}$-system $\mathbf{X}=(X, X, \mu, T)$ can be represented as a minimal topologically distal system $(X, T, \mu)$ with $\mu \in M_{T}^{\mathrm{erg}}(X)$.

This topological model need not, in general, be uniquely ergodic. In other words there are measure distal systems for which no uniquely ergodic topologically distal model exists.

13.5. Proposition. 1. There exists an ergodic non-Kronecker measure distal system $(\Omega, \mathcal{F}, m, T)$ with nontrivial maximal Kronecker factor $\left(\Omega_{0}, \mathcal{F}_{0}, m_{0}, T\right)$ such that $(i)$ the extension $(\Omega, \mathcal{F}, m, T) \rightarrow\left(\Omega_{0}, \mathcal{F}_{0}, m_{0}, T\right)$ is finite to one a.e. and (ii) every nontrivial factor map of $\left(\Omega_{0}, \mathcal{F}_{0}, m_{0}, T\right)$ is finite to one.

2. A system $(\Omega, \mathcal{F}, m, T)$ as in part 1 does not admit a topologically distal strictly ergodic model.

Proof. 1. Irrational rotations of the circle as well as adding machines are examples of Kronecker systems satisfying condition (ii). There are several constructions in the literature of ergodic, non-Kronecker, measure distal, two point extensions of these Kronecker systems. A well known explicit example is the strictly ergodic Morse minimal system.

2. Assume to the contrary that $(X, \mu, T)$ is a distal strictly ergodic model for $(\Omega, \mathcal{F}, m, T)$. Let $(Z, T)$ be the maximal equicontinuous factor of $(X, T)$ and let $\eta$ be the unique invariant probability measure on $Z$. Since by assumption $(X, \mu, T)$ is not Kronecker it follows that $\pi: X \rightarrow Z$ is not one to one. By Furstenberg's structure theorem for minimal distal systems $(Z, T)$ is nontrivial and moreover there exists an intermediate extension $X \rightarrow Y \stackrel{\sigma}{\rightarrow} Z$ such that $\sigma$ is an isometric extension. A well known construction implies the existence of a minimal group extension $\rho:(\tilde{Y}, T) \rightarrow$ $(Z, T)$, with compact fiber group $K$, such that the following diagram is commutative (see Section 5 above). We denote by $\nu$ the unique invariant measure on $Y$ (the image of $\mu$ ) and let $\tilde{\nu}$ be an ergodic measure on $\tilde{Y}$ which projects onto $\nu$. The dotted arrows denote measure theoretic factor maps.

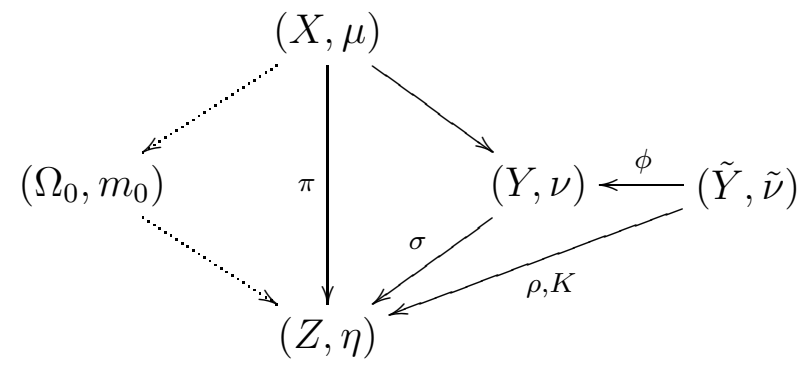


Next form the measure $\theta=\int_{K} R_{k} \tilde{\nu} d m_{K}$, where $m_{K}$ is Haar measure on $K$ and for each $k \in K, R_{k}$ denotes right translation by $k$ on $\tilde{Y}$ (an automorphism of the system $(\tilde{Y}, T))$. We still have $\phi(\theta)=\nu$.

A well known theorem in topological dynamics (see [74]) implies that a minimal distal finite to one extension of a minimal equicontinuous system is again equicontinuous and since $(Z, T)$ is the maximal equicontinuous factor of $(X, T)$ we conclude that the extension $\sigma: Y \rightarrow Z$ is not finite to one. Now the fibers of the extension $\sigma$ are homeomorphic to a homogeneous space $K / H$, where $H$ is a closed subgroup of $K$. Considering the measure disintegration $\theta=\int_{Z} \theta_{z} d \eta(z)$ of $\theta$ over $\eta$ and its projection $\nu=\int_{Z} \nu_{z} d \eta(z)$, the disintegration of $\nu$ over $\eta$, we see that a.e. $\theta_{z} \equiv m_{K}$ and $\nu_{z} \equiv m_{K / H}$. Since $K / H$ is infinite we conclude that the measure theoretical extension $\sigma:(Y, \nu) \rightarrow(Z, \eta)$ is not finite to one. However considering the dotted part of the diagram we arrive at the opposite conclusion. This conflict concludes the proof of the proposition.

In 68. Ornstein and Weiss introduced the notion of tightness for measure preserving systems and the analogous notion of mean distality for topological systems.

13.6. Definition. Let $(X, T)$ be a topological system.

1. A pair $(x, y)$ in $X \times X$ is mean proximal if for some (hence any) compatible metric $d$

$$
\limsup _{n \rightarrow \infty} \frac{1}{2 n+1} \sum_{i=-n}^{n} d\left(T^{i} x, T^{i} y\right)=0 .
$$

If this limsup is positive the pair is called mean distal.

2. The system $(X, T)$ is mean distal if every pair with $x \neq y$ is mean distal.

3. Given a $T$-invariant probability measure $\mu$ on $X$, the triple $(X, \mu, T)$ is called tight if there is a $\mu$-conull set $X_{0} \subset X$ such that every pair of distinct points $(x, y)$ in $X_{0} \times X_{0}$ is mean distal.

Ornstein and Weiss show that tightness is in fact a property of the measure preserving system $(X, \mu, T)$ (i.e. if the measure system $(X, \mathcal{X}, \mu, T)$ admits one tight model then every topological model is tight). They obtain the following results.

\subsection{Theorem.}

1. If the entropy of $(X, \mu, T)$ is positive and finite then $(X, \mu, T)$ is not tight.

2. There exist strictly ergodic non-tight systems with zero entropy.

Surprisingly the proof in 68] of the non-tightness of a positive entropy system does not work in the case when the entropy is infinite which is still open.

J. King gave an example of a tight system with a non-tight factor. Following this he and Weiss 68 established the following result. Note that this theorem implies that tightness and mean distality are not preserved by factors.

13.8. Theorem. If $(X, X, \mu, T)$ is ergodic with zero entropy then there exists a meandistal system $(Y, \nu, S)$ which admits $(X, X, \mu, T)$ as a factor. 


\section{REFERENCES}

[1] J. Aaronson and B. Weiss, Remarks on tightness of cocycles, Collloq. Math. 84/85, Part $2,(2000), 363-376$.

[2] R. L. Adler, A. G. Konheim and M. H. McAndrew, Topological entropy, Trans. Amer. Math. Soc. 114, (1965), 309-319.

[3] E. Akin, Recurrence in topological dynamics, Furstenberg families and Ellis actions, Plenum Press, New York and London, 1997.

[4] E. Akin and E. Glasner, Topological ergodic decomposition and homogeneous flows, AMS Contemporary Math. 215, (1998), 43-52.

[5] _ Residual properties and almost equicontinuity, J. d'Analyse Math. 84, (2001), 243-286.

[6] L. Auslander, L. Green and F. Hahn, Flows on homogeneous spaces, Annals of mathematics studies 53, Princeton University Press, Princeton, New Jersey, 1963.

[7] A. Bellow and H. Furstenberg, An application of number theory to ergodic theory and the construction of uniquely ergodic models, Israel J. of Math. 33, (1979), 231-240.

[8] F. Blanchard, Fully positive topological entropy and topological mixing, Contemp. Math. Symbolic Dynamics and Applications 135, Amer. Math. Soc. , Providence, 1992, pp. 95105.

[9] —_ A disjointness theorem involving topological entropy, Bull. de la Soc. Math. de France 121, (1993), 465-478.

[10] F. Blanchard, E. Glasner and B. Host, A variation on the variational principle and applications to entropy pairs, Ergod. Th. Dynam. Sys. 17, (1997), 29-43.

[11] F. Blanchard, E. Glasner, S. Kolyada and A. Maass, On Li-Yorke pairs, J. für die Reine und Angewandte Mathematik 547, (2002), 51-68.

[12] F. Blanchard, B. Host, A. Maass, S. Martínez and D. Rudolph, Entropy pairs for a measure, Ergod. Th. Dynam. Sys. 15, (1995), 621-632.

[13] F. Blanchard, B. Host and A. Maass, Topological complexity, Ergod. Th. Dynam. Sys. 20, (2000), 641-662.

[14] F. Blanchard, B. Host and S. Ruette, Asymptotic pairs in positive-entropy systems, Ergod. Th. Dynam. Sys. 22, (2002), 671-686.

[15] M. Boyle and T. Downarowicz, The entropy theory of symbolic extensions, Inventiones Mathematicae, 156, (2004), 119-161.

[16] R. V. Chacón, Weakly mixing transformations which are not strongly mixing, Proc. Amer. Math. Soc. 22, (1969), 559-562.

[17] M. Denker, C. Grillenberger and K. Sigmund, Ergodic theory on compact spaces, Lecture Notes in Math. 527, Springer-Verlag, 1976.

[18] T. Downarowicz, The Choquet simplex of invariant measures for minimal flows, Israel J. of Math. 74, (1991), 241-256.

[19] T. Downarowicz and Y. Lacroix, Almost 1-1 extensions of Furstenberg-Weiss type and applications to Toeplitz flows, Studia Math. 130, (1998), 149-170.

[20] T. Downarowicz and J. Serafin, Possible entropy functions, Israel J. Math. 135, (2003), 221-250

[21] E. G. Effros, Transformation groups and $C^{*}$-algebras, Ann. of Math. 81, (1976), 38-55.

[22] H. Furstenberg, The structure of distal flows, American J. of Math. 85, (1963), 477-515.

[23] _ Disjointness in ergodic theory, minimal sets, and a problem in Diophantine approximation, Math. System Theory 1, (1967), 1-49.

[24] _ Ergodic behavior of diagonal measures and a theorem of Szemerédi on arithmetic progressions, J. d'Analyse Math. 31, (1977), 204-256.

$[25]$ _ Recurrence in ergodic theory and combinatorial number theory, Princeton university press, Princeton, N.J., 1981.

[26] H. Furstenberg and B. Weiss, Topological dynamics and combinatorial number theory, J. d'Analyse Math. 34, (1978), 61-85.

[27] _ The finite multipliers of infinite transformation, Spriger Verlag Lecture Notes in Math. 688, (1978), 127-132. 
[28] - On almost 1-1 extensions, Israel J. Math. 65, (1989), 311-322.

[29] R. Ellis, E. Glasner and L. Shapiro, Proximal-isometric flows, Advances in Math. 17, (1975), $213-260$.

[30] T. Giordano, I. F. Putnam, and C. F. Skau, Topological orbit equivalence and $C^{*}$-crossed products, J. reine angew. Math. 469, (1995), 51-111.

[31] E. Glasner, A simple characterization of the set of $\mu$-entropy pairs and applications, Israel J. of Math. 102, (1997), 13-27.

[32] _ On minimal actions of Polish groups, Topology and Its Applications, 85, (1998), 119-125.

[33] Structure theory as a tool in topological dynamics, Descriptive set theory and dynamical systems, LMS Lecture note Series 277, Cambridge University Press, Cambridge, 2000, 173-209.

[34] Topics in topological dynamics, 1991 to 2001. Recent progress in general topology, II, North-Holland, Amsterdam, 2002, 153-175.

[35] — Ergodic theory via joinings, AMS, Surveys and Monographs, 101, 2003.

[36] E. Glasner, B. Host and D. Rudolph, Simple systems and their higher order self-joinings, Israel J. of Math. 78, (1992), 131-142.

[37] E. Glasner and D. Maon, Rigidity in topological dynamics, Ergod. Th. Dynam. Sys. 9, (1989), 309-320.

[38] E. Glasner and B. Weiss, Minimal transformations with no common factor need not be disjoint, Israel J. of Math. 45, (1983), 1-8.

[39] _ Sensitive dependence on initial conditions, Nonlinearity 6, (1993), 1067-1075.

[40] Strictly ergodic, uniform positive entropy models, Bull. Soc. Math. France 122, (1994), 399-412.

[41] _ Topological entropy of extensions, Proceedings of the 1993 Alexandria conference, Ergodic theory and its connections with harmonic analysis, Editors: K. E. Petersen and I. A. Salama, LMS Lecture note Series 205, Cambridge University Press, Cambridge, 1995, 299-307.

[42] J. Glimm, Locally compact transformation groups, Trans. Amer. Math. Soc. 101, (1961), 124-138.

[43] T. N. T. Goodman, Relating topological entropy with measure theoretic entropy, Bull. London. Math. Soc. 3, (1971), 176-180.

[44] F. Hahn and Y. Katznelson, On the entropy of uniquely ergodic transformations, Trans. Amer. Math. Soc. 126, (1967), 335-360.

[45] J. Hansel and J.-P. Raoult, Ergodicity, uniformity and unique ergodicity, Indiana. Univ. Math. J. 23, (1974), 221-237.

[46] B. Hasselblatt and A. Katok, Principle structures, Handbook of Dynamical Systems, Vol. 1A, Hasselblatt and Katok, eds, Elsevier, Amsterdam, (2002), 1-203.

[47] B. Host, Mixing of all orders and independent joinings of systems with singular spectrum, Israel J. Math. 76, (1991), 289-298.

[48] W. Huang and X. Ye, An explicit scattering, non-weakly mixing example and weak disjointness, Nonlinearity 15, (2002), 849-862.

[49] , Topological complexity, return times and weak disjointness, to appear in Ergod. Th. Dynam. Sys. .

[50] R. I. Jewett, The prevalence of uniquely ergodic systems, J. Math. Mech. 19, (1970), 717729.

[51] A. del Junco, M. Lemańczyk and M. K. Mentzen, Semisimplicity, joinings and group extensions, Studia Math. 112, (1995), 141-164.

[52] A. del Junco, M. Rahe and L. Swanson, Chacón's automorphism has minimal self-joinings, J. d'Analyse Math. 37, (1980), 276-284.

[53] A. del Junco and D.J. Rudolph, On ergodic actions whose self-joinings are graphs, Ergod. Th. Dynam. Sys. 7, (1987), 531-557.

[54] B. Kamiński, A. Siemaszko and J. Szymański, The determinism and the Kolmogorov property in topological dynamics, Preprint. 
[55] J. W. Kammeyer and D. Rudolph, Restricted orbit equivalence for actions of discrete amenable groups, Cambridge tracts in mathematics 146, Cambridge University Press, Cambridge, 2002.

[56] Y. Katznelson and B. Weiss, When all points are recurrent/generic, Ergodic theory and dynamical systems I, Proceedings, Special year, Maryland 1979-80, Birkhäuser, Boston, 1981.

[57] H. B. Keynes and J. B. Robertson, Eigenvalue theorems in topological transformation groups, Trans. Amer. Math. Soc. 139, (1969), 359-369.

[58] J. King, Ergodic properties where order 4 implies infinite order, Israel J. of Math. 80, (1992), 65-86.

[59] W. Krieger, On unique ergodicity, Proc. sixth Berkeley symposium Math. Statist. Probab. Univ. of California Press, 1970, 327-346.

[60] I. Kriz, Large independent sets in shift-invariant graphs. Solution of Bergelson's problem, Graphs and combinatorics, 3, (1987), 145-158.

[61] E. Lehrer, Topological mixing and uniquely ergodic systems, Israel J. of Math. 57, (1987), 239-255.

[62] M. Lemańczyk, F. Parreau and J.-P. Thouvenot, Gaussian automorphisms whose ergodic self-joinings are Gaussian, Fund. Math. 164, (2000), 253-293.

[63] E. Lindenstrauss, Lowering topological entropy, J. d'Analyse Math. 67, (1995), 231-267.

[64] _ Measurable distal and topological distal systems, Ergod. Th. Dynam. Sys. 19, (1999), 1063-1076.

[65] D. C. McMahon, Weak mixing and a note on the structure theorem for minimal transformation groups Illinois J. of Math. 20 (1976), 186-197.

[66] _ Relativized weak disjointness and relative invariant measures, Trans. Amer. Math. Soc. 236, (1978), 225-137.

[67] N. S. Ormes, Strong orbit realization for minimal homeomorphisms, J. Anal. Math. 71, (1997), 103-133.

[68] D. Ornstein and B. Weiss, Mean distality and tightness. To appear in the Proceedings of the Steklov Mathematical Institute.

[69] W. Parry, Zero entropy of distal and related transformations, in Topological dynamics, J. Auslader and W. Gottschalk, eds., Benjamin, New York, 1967.

[70] K. Petersen, Disjointness and weak mixing of minimal sets, Proc. Amer. Math. Soc. 24, (1970), 278-280.

[71] M. Ratner, Horocycle flows, joinings and rigidity of products, Annals of Math. 118, (1983), 277-313.

[72] D. J. Rudolph, An example of a measure-preserving transformation with minimal selfjoinings and applications, J. d'Analyse Math. 35, (1979), 97-122.

[73] V.V. Ryzhikov, Joinings, intertwining operators, factors and mixing properties of dynamical systems, Russian Acad. Izv. Math. 42, (1994), 91-114.

[74] R. Sacker and G. Sell, Finite extensions of minimal transformation groups, Trans. Amer. Math. Soc. 190, (1974), 325-334.

[75] M. Shub and B. Weiss, Can one always lower topological entropy?, Ergod. Th. Dynam. Sys. 11 (1991), 535-546.

[76] S. D. Silvestrov and J. Tomiyama, Topological dynamical systems of type I, Expo. Math. 20, (2002), 117-142.

[77] J.-P. Thouvenot, Some properties and applications of joinings in ergodic theory, Proceedings of the 1993 Alexandria conference, Ergodic theory and its connections with harmonic analysis, Editors: K. E. Petersen and I. A. Salama, LMS Lecture note Series 205, Cambridge University Press, Cambridge, 1995, 207-238.

[78] W. A. Veech, The equicontinuous structure relation for minimal Abelian transformation groups, Amer. J. of Math. 90, (1968), 723-732.

[79] — Point distal flows, Amer. J. of Math. 92, (1970), 205-242.

[80] —_, Topological dynamics, Bull. Amer. Math. Soc. 83, (1977), 775-830.

[81] _ _ A criterion for a process to be prime, Monatsh. Math. 94, (1982), 335-341. 
[82] B. Weiss, Measurable dynamics, Contemporary Mathematics 26, Conference in Modern Analysis and Probability, (1984), 395-421.

[83] — Countable generators in dynamics - Universal minimal models, Contemporary Mathematics 94, (1989), 321-326.

[84] _ Multiple recurrence and doubly minimal systems, Contemporary Mathematics 215, (1998), 189-196.

[85] _ A survey of generic dynamics, In Descriptive set theory and dynamical systems, LMS Lecture note Series 277, Cambridge University Press, 2000, 273-291.

[86] _ Single orbit dynamics, CBMS, Regional Conference Series in Math. 95, Amer. Math. Soc. Providence RI, 2000.

[87] R. J. Zimmer, Extensions of ergodic group actions, Illinois J. Math. 20, (1976), 373-409.

[88] _ Ergodic actions with generalized discrete spectrum, Illinois J. Math. 20, (1976), $555-588$.

Department of Mathematics, Tel Aviv University, Tel Aviv, Israel

E-mail address: glasner@math.tau.ac.il

Institute of Mathematics, Hebrew University of Jerusalem, Jerusalem, Israel

E-mail address: weiss@math.huji.ac.il 